\title{
TARPs Modulate Receptor-Mediated Paired-Pulse Depression and Recovery from Desensitization
}

\author{
Suma Priya Sudarsana Devi, ${ }^{1,3}$ Yiru Cheng, ${ }^{4}$ Susumu Tomita, ${ }^{2}$ James R. Howe, ${ }^{1 \dagger}$ and ${ }^{\circledR}$ Wei Zhang ${ }^{4}$ \\ ${ }^{1}$ Department of Pharmacology, Yale University School of Medicine, New Haven, Connecticut 06520, ${ }^{2}$ Department of Cellular and Molecular \\ Physiology, Department of Neuroscience, and Kavli Institute for Neuroscience, Yale University School of Medicine, New Haven, Connecticut 06520, \\ ${ }^{3}$ Department of Surgery, University of Alabama at Birmingham, Birmingham, Alabama 35233, and ${ }^{4}$ Department of Pharmacology of Chinese \\ Materia Medica, Institution of Chinese Integrative Medicine, Hebei Medical University, Shijiazhuang, Hebei Province 050017, China
}

Transmembrane AMPA receptor regulatory proteins (TARPs) are auxiliary AMPA receptor subunits that play a key role in receptor trafficking and in modulating receptor gating. The ability of TARPs to slow both deactivation and desensitization is isoform specific. However, TARP isoform-specific modulation of receptor properties remains uncharacterized. Here, we compare the isoform-specific effects of $\gamma-2, \gamma-3, \gamma-4$, and $\gamma-8$ TARPs on recovery from desensitization and responses to pairs of brief applications of glutamate. All four isoforms were able to reduce receptor-mediated paired-pulse depression and significantly speed recovery from desensitization in an isoform-specific manner. In the presence of TARPs, recovery time courses were observed to contain two components, fast and slow. The proportion of fast and slow components was determined by the TARP isoform. The time constant of recovery was also altered by the duration of glutamate application. When studies with TARP chimeras were performed, TARP extracellular loops were found to play a vital role in TARP modulation of recovery. Thus, isoform-specific differences in TARP modulation of recovery from desensitization influence receptor responses to repeated brief applications of glutamate, and these differences may impact frequency-dependent synaptic signaling in the mammalian central nervous system.

Key words: AMPA receptor; glutamate; kinetics; modulation; TARPs

Significance Statement

AMPA receptors are major determinants of excitatory synaptic strength. The channel kinetics of AMPA receptors contribute to the kinetics of synaptic transmission. Transmembrane AMPA receptor regulatory proteins (TARPs) auxiliary subunits can modulate the decay kinetics of AMPA receptors. However, whether TARP isoforms specifically modulate receptor recovery is unclear. Here, we investigated the recovery kinetics of AMPA receptors by expressing various TARP isoforms and chimeras. We observed that the TARP isoforms and duration of glutamate application uniquely modulate time constants and the proportion of fast and slow components through a previously unidentified TARP domain. Given the impact of recovery kinetics on receptor responses to repetitive stimulation such as synaptic transmission, this work will be of great interest in the field of excitatory synaptic transmission research.

Received Dec. 21, 2019; revised Aug. 7, 2020; accepted Sep. 14, 2020.

Author contributions: J.R.H., S.T. and W.Z. designed research; S.P.S.D., Y.C., and W.Z. performed research;

S.P.S.D., S.T., J.R.H., and W.Z. analyzed data; S.P.S.D., S.T., J.R.H., and W.Z. wrote the paper.

${ }^{\dagger}$ Deceased, March 8, 2019.

This work was supported by the National Institutes of Health (NIH) Grant NS057725 (to J.R.H.), funds from Yale University (to J.R.H.), National Natural Science Foundation of China Grants 81872848 and 81573416 (to W.Z.), National Major Special Project on New Drug Innovation Grant 2018ZX09711001-004-003 (to W.Z.), the Jing-Jin-Ji Basic Research Cooperation Project H2018206641 (to W.Z.), High talent science research project of Hebei Province A201701010068 (to W.Z.), and the NIH Grant MH077939 (to S.T.). We thank Dr. Claudia Schmauss for critically reading our manuscript.

The authors declare no competing financial interests.

Correspondence should be addressed Susumu Tomita at susumu.tomita@yale.edu or Wei Zhang at weizhang@hebmu.edu.cn.

https://doi.org/10.1523/JNEUROSCI.3026-19.2020

Copyright $\odot 2020$ the authors

\section{Introduction}

AMPA receptors are glutamate-gated ion channels that mediate fast excitatory synaptic transmission in the mammalian CNS (Hollmann and Heinemann, 1994; Dingledine et al., 1999; Erreger et al., 2004; Traynelis et al., 2010). The core AMPA receptor is formed from homomeric or heteromeric assemblies of subunits, GluA1-4. Each of these subunits has an N-terminal domain (ATD), a ligand-binding domain (LBD), a transmembrane domain (TMD), and an intracellular C terminus (Traynelis et al., 2010; Sobolevsky, 2015). A total of four core subunits are needed to form a functional receptor which recapitulates many of the properties of native AMPA receptors. However, these receptors also have auxiliary subunits which modulate receptor trafficking and their biophysical and kinetic behaviors (Coombs and Cull- 
Candy, 2009; Schwenk et al., 2009; Kato et al., 2010; Jackson and Nicoll, 2011; Schwenk et al., 2012; Shanks et al., 2012; Yan and Tomita, 2012; McGee et al., 2015).

Stargazin (TARP $\gamma-2$ ) was the first auxiliary subunit identified for the AMPA receptor, and it was subsequently shown to be a member of the transmembrane AMPA receptor regulatory protein (TARP) family of proteins (Hashimoto et al., 1999; Chen et al., 2000; Bredt and Nicoll, 2003; Tomita et al., 2003). We, and others, have shown that TARPs modulate receptor responses to both brief and sustained applications of glutamate in an isoformspecific manner (Tomita et al., 2005; Turetsky et al., 2005; Cho et al., 2007; Körber et al., 2007; Kott et al., 2007; Milstein et al., 2007; Milstein and Nicoll, 2009; Jackson et al., 2011). There are six TARP isoforms, four Type I TARPs $(\gamma-2, \gamma-3, \gamma-4$, and $\gamma-$ $8)$ and two Type II TARPs ( $\gamma-5$ and $\gamma-7)$. Type I TARPs potentiate all AMPAR subunits, while Type II TARPs modulates specific combinations of subunits (Kato et al., 2008). In addition, Type I TARPs have both intracellular $\mathrm{N}$ and $\mathrm{C}$ termini. Their ability to slow deactivation and desensitization decays of AMPA receptors appears to depend primarily on sequence elements which are contained within a large extracellular loop (Ex1) between the first and second of four transmembrane helices (Tomita et al., 2004, 2005; Cho et al., 2007; Milstein et al., 2007).

The distribution of Type I TARPs in the CNS is isoform-dependent. For example, $\gamma-2$ is the primary TARP expressed in the cerebellum, $\gamma-3$ and $\gamma-8$ are expressed mainly in the cerebral cortex and hippocampus, respectively, whereas $\gamma-4$ is diffusely expressed throughout the brain (Tomita et al., 2003; Fukaya et al., 2005). Because of quantitative differences in the effect of each TARP isoform on receptor gating, it is hypothesized that regional differences in TARP expression contribute to synapse-specific differences in the kinetics of EPSCs.

Here, we compare the effects of all four Type I TARP isoforms $(\gamma-2, \gamma-3, \gamma-4$, and $\gamma-8)$ on recovery from desensitization and receptor responses to pairs of brief applications of glutamate. Our results show that different TARPs have distinct effects on the time course of recovery, and that these differences result from isoform-specific differences in TARP modulation of receptor responses to repeated brief applications of glutamate. Sequence elements in the second extracellular loop (Ex2) between TM3 and TM4 of Type I TARPs also appear to be the primary determinants of isoform-specific differences in TARP modulation of recovery from desensitization.

\section{Materials and Methods}

Cell culture and transfection

As previously described, tsA201 cells were maintained and transfected with a flip splice variant of a GluA1 plasmid (Robert et al., 2001). Individual GluA1 and TARP clones were co-transfected at ratios of 1:1-1:3.

\section{Chimeras construct generation}

PCR was used to construct chimeras with the first extracellular domain (Ex1) exchanged between TM1 and TM2 (D31 to S105 for $\gamma-2$ and D31 to S108 for $\gamma-4)$ and between $\gamma-2$ and $\gamma-4$ to create $\gamma-2(\gamma-4 \operatorname{Ex} 1)$ and $\gamma-4(\gamma-2 \mathrm{Ex} 1)$. PCR was also used to create tandem constructs with the $\mathrm{C}$ terminus of GluA1 directly fused to the $\mathrm{N}$ terminus of either $\gamma-2$ or $\gamma-3$ (Cho et al., 2007; Morimoto-Tomita et al., 2009). Chimeras with the second extracellular domain (Ex2) between TM3 and TM4 (from V155 to Y181 in $\gamma-2$ and V158 to Y186 in $\gamma-4)$ interchanged between $\gamma-2$ and $\gamma-4$ were also constructed by performing overlap extension PCR to create $\gamma-2(\gamma-4 \operatorname{Ex} 2)$ and $\gamma-4(\gamma-2 \mathrm{Ex} 2)$, as well as double chimeras with both extracellular domains swapped between $\gamma-2$ and $\gamma-4$ to create $\gamma-$ $2(\gamma-4 \operatorname{Ex} 1 \mathrm{Ex} 2)$ and $\gamma-4(\gamma-2 \operatorname{Ex} 1 \mathrm{Ex} 2)$. The final constructs were verified by DNA sequencing. Nucleotide sequences of the two sets of primer pairs used for the construction of $\gamma-2(\gamma-4 \operatorname{Ex} 2)$ and $\gamma-2(\gamma-4 \operatorname{EX} 1 \operatorname{Ex} 2)$ are the following:

5'-GCTCTAGAATGGGGCTGTTTGATCG-3'

$5^{\prime}$-CCCAAGCTTTCAAACGGGAGTCG-3'

5'-GACAAGCGTGACGAAGACAAGAAGAACCATTACAACTAC GGCTGGTCTTTTTACTTCGGGGCCCTGTCCTTC-3'

$5^{\prime}$-CTTGTCTTCGTCACGCTTGTCACTGGGGTCGCCCGTGTT GCTGGAAATGTAGACGATGATCCCGATGATATTACTAAGAC-3'

The chimeras, $\gamma-4(\gamma-2 \mathrm{Ex} 2)$ and $\gamma-4(\gamma-2 \mathrm{EX} 1 \mathrm{Ex} 2)$, were constructed by using the following primers:

5'-GCTCTAGAATGGTGCGATGCGAC-3'

5'-CCCAAGCTTTCAAACGGGAGTCG-3' 5' $^{\prime}$-CTCCAAGAGTG ACTCCAAAAAGAACAGCTACTCCTACGGCTGGTCCTTCTACTT TGGAGCCCTGTCGTTTATTG-3'

5'-CTTTTTGGAGTCACTCTTGGAGGGGTCTCCAGCATTGGC TGATATATACACGATGATGCCGATGATATTACTG-3'.

\section{Electrophysiology}

Recordings from outside-out patches were performed 1-3 d posttransfection at room temperature with an EPC-9 amplifier (HEKA) as previously described (Robert and Howe, 2003). The holding potential was $100 \mathrm{mV}$. The external solution used was composed of the following: 150 $\mathrm{mm} \mathrm{NaCl}, 3 \mathrm{~mm} \mathrm{KCl}, 2 \mathrm{~mm} \mathrm{CaCl}$, $1 \mathrm{~mm} \mathrm{MgCl}_{2}, 5 \mathrm{~mm}$ glucose, and 10 mM HEPES (pH 7.4). Patch pipettes (open tip resistance, 3-8 $\mathrm{M} \Omega$ ) were filled with a solution containing the following: $135 \mathrm{~mm} \mathrm{CsF}, 33 \mathrm{~mm}$ $\mathrm{CsOH}, 2 \mathrm{~mm} \mathrm{MgCl}$, 1 mm CaCl 2,11 mm EGTA, and $10 \mathrm{~mm} \mathrm{HEPES} \mathrm{(pH}$ 7.4), for all experiments (Figure 1 and others) except those represented in Figure 2 (which used a KF-based internal solution). No obvious differences were observed between use of the two internal solutions in our experiments.

Glutamate was added to the external solution and was applied with theta glass pipettes mounted on a piezoelectric bimorph (Robert and Howe, 2003). The $10-90 \%$ rise times of agonist-evoked currents typically ranged from 0.4 to $0.6 \mathrm{~ms}$, and the rate of solution exchange (estimated from open-tip potentials) ranged from 100 to $200 \mu$ s. The bath was superfused constantly with normal external solution flowing at a rate of $1 \mathrm{ml} / \mathrm{min}$.

Agonist-evoked currents recorded in outside-out patches were ana$\log$ low-pass filtered at $3 \mathrm{kHz}$ and written directly to the hard drive of the computer at sampling rates of $10-20 \mathrm{kHz}$. Recovery data were obtained from two-pulse protocols. The peak amplitude of the current evoked by the second pulse was expressed as a fraction of the response to the paired first pulse. Mean results from several patches were fitted with double Hodgkin-Huxley-type (H-H-type) equations as described elsewhere (Robert and Howe, 2003; Erreger et al., 2004; Zhang et al., 2006).

\section{Statistics and data analysis}

All analyses were performed using IgorPro software (Wavemetrics). For statistical analyses, the Shapiro-Wilkes test was used for normality comparisons. After normality was confirmed, a two-tailed Student's $t$ test or one-way ANOVA followed by Bonferroni's post hoc test and two-way ANOVA were used for statistical comparisons; $p<0.05$ was considered significant.

\section{Results}

Differences in TARP modulation have been noted between different GluA subunits (Turetsky et al., 2005; Kott et al., 2007), yet the main features of TARP modulation of AMPA-receptor gating are similar (Cho et al., 2007). For GluA1 receptors, they recover more slowly from desensitization in the absence of TARPs than receptors formed by other GluA subunits (Robert and Howe, 2003; Robert et al., 2005). Consequently, this slower recovery of GluA1 receptors facilitates detection and quantification of increases in their rate of recovery. Therefore, GluA1 was selected for GluA/TARP co-expression studies in which the ability of TARP isoforms to speed recovery from desensitization was examined. 


\section{TARP modulation of paired-pulse responses}

Paired-pulse stimulation is widely used to examine repetitive responses to presynaptic glutamate release. These responses are affected not only by presynaptic regulation, but also by postsynaptic regulation. When brief applications of glutamate are repeated with short intervals, the amplitude of the peak currents declines along the time course the current would have followed if glutamate had been applied continuously (Raman and Trussell, 1995). The envelope of peak currents evoked at short intervals matches the desensitization time course because AMPA-receptor activation and desensitization proceed in parallel from a common set of short-lived closed states (Patneau and Mayer, 1991; Vyklicky et al., 1991; Raman and Trussell, 1992, 1995; Jonas et al., 1993; Partin et al., 1996; Robert and Howe, 2003). Notably, AMPA-receptor activation and desensitization proceed even after glutamate is absent from the external solution. We previously demonstrated that TARP isoforms $\gamma-2$ and $\gamma-8$ both blunt the decline of GluA1 currents in response to a train of 2-ms applications of glutamate (Cho et al., 2007). Moreover, this modulation occurred when both short and long interpulse intervals were used. To further study this modulation in the present study, we co-expressed each TARP isoform with GluA1 in tsA201 cells and performed outside-out patch recordings. Briefly, after a 2-ms application of $10 \mathrm{~mm}$ glutamate was applied by a piezo electric device, paired-pulse data were collected for each of the four Type I TARPs at interpulse intervals ranging from 10 to $500 \mathrm{~ms}$ (Fig. 1).

Each of the four Type I TARPs reduced receptor-mediated paired-pulse depression with $10 \mathrm{~ms}$ intervals (Fig. 1A). These short interval data are consistent with previous evidence that all four TARPs induce a slower fade of current during a sustained application of glutamate, thereby reflecting a slower entry of receptors into a desensitized state (Priel et al., 2005; Tomita et al., 2005; Turetsky et al., 2005; Cho et al., 2007; Milstein et al., 2007). TARPs slow deactivation and desensitization fade by selectively speeding receptor activation, resulting in a 2 - to 4 -fold increase in the average number of openings that occur before the receptor desensitizes (Tomita et al., 2005; Cho et al., 2007; Zhang et al., 2014; Howe, 2015; Carbone and Plested, 2016). TARP isoforms have previously been characterized according to their modulation of desensitization (and deactivation). For example, TARPs $\gamma-2$ and $\gamma-3$ produce a similar slowing of desensitization, whereas TARPs $\gamma-4$ and $\gamma-8$ exhibit 2-fold slower desensitization (Cho et al., 2007; Milstein et al., 2007). Therefore, we hypothesized that $\gamma-2$ and $\gamma-3$ would produce similar effects on receptor-mediated paired-pulse depression, and that the reductions observed with these two isoforms would be less than the reductions observed with $\gamma-4$ and $\gamma-8$ (which would also be similar to each other). This hypothesis was confirmed with $\gamma-2$ and $\gamma-3$, and $\gamma-4$ and $\gamma-8$, exhibiting similar paired-pulse depression for each pair, and the former reduction being less than the latter (Fig. 1B). Thus, TARP isoforms appear to exhibit distinct recovery kinetics.

\section{TARP modulation of recovery from desensitization}

Rate of recovery from desensitization also influences receptormediated paired-pulse depression. Previous reports have noted that $\gamma-2$ speeds recovery and destabilizes the desensitized states of the receptor (Priel et al., 2005; Turetsky et al., 2005). In addition, we have shown that $\gamma-2$ and $\gamma-8$ reduce paired-pulse depression in response to brief applications of glutamate at interpulse intervals which are too long to be influenced by the rate at which the receptors desensitize (Cho et al., 2007).
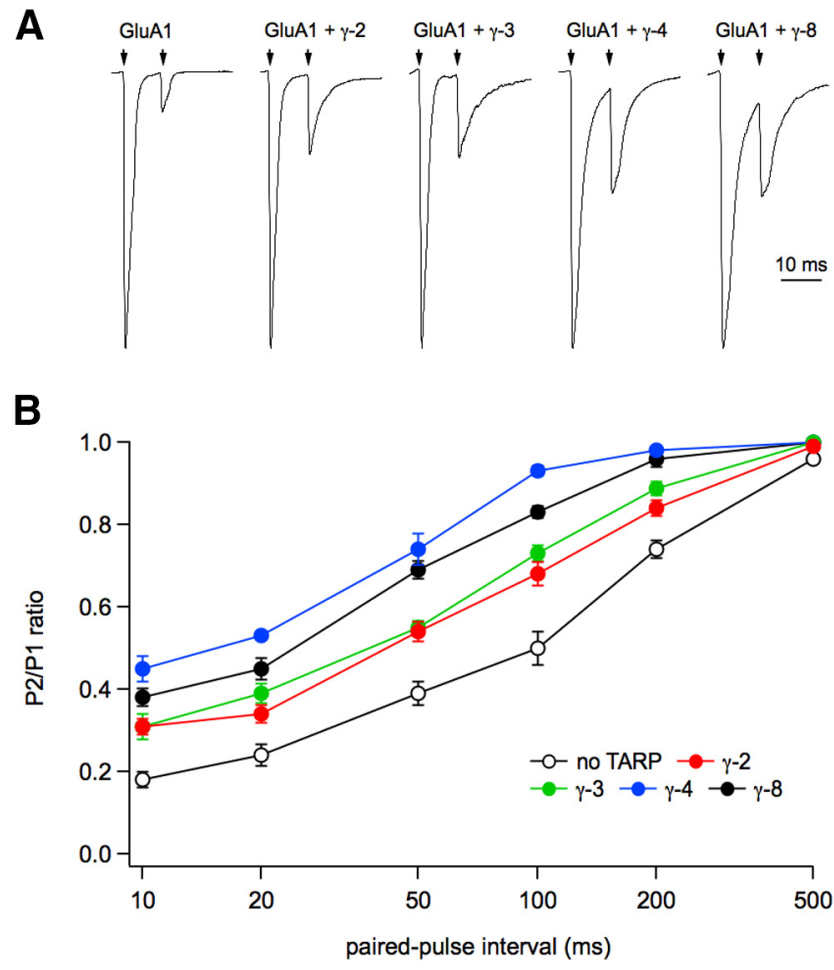

Figure 1. The effects of all Type I TARPs on receptor-mediated paired-pulse depression. A, Responses to brief ( $2 \mathrm{~ms}$ ) pulses of $10 \mathrm{~mm}$ glutamate applied (indicated with arrows) at an interval of $10 \mathrm{~ms}$ to individual outside-out patches from cells expressing GluA1 alone or cells co-transfected with GluA1 and the indicated Type I TARP. B, Results for receptor-mediated paired-pulse depression at various interpulse intervals. Two brief (2 ms) applications of $10 \mathrm{~mm}$ glutamate were made at intervals varying from 10 to $500 \mathrm{~ms}$ to patches from cells expressing GluA1 alone (no TARP) or co-expressing GluA1 with the indicated Type I TARP. The results (mean \pm SEM, $n=5-9$ per data point) are expressed as the ratio of the peak amplitude (measured from baseline) of the second and first responses (P2/P1). The mean values obtained at each interval were compared with one-way ANOVA. At all of the intervals tested, the mean value for GluA1 alone (without TARP) significantly differed from the corresponding values for GluA1 expressed with each of the four TARPs (20 comparisons: $p<0.05$ for A1 vs A1 coexpressed with $\gamma-2$ at 20 and $200 \mathrm{~ms}, p<0.01$ for the 18 remaining comparisons). For the interpulse intervals, the $\gamma-2$ and $\gamma-3$ values significantly differed from the $\gamma-4$ values at all of the intervals $(p<0.05$ at $200 \mathrm{~ms}$ for $\gamma-3 ; p<0.01$ for the other nine comparisons) and from the $\gamma-8$ value at the 50 -ms interval $(p<0.05)$. The $\gamma-2$ and $\gamma-8$ values also significantly differed at the intervals ranging from 20 to $200 \mathrm{~ms}$ $(p<0.01)$. The values obtained for GluA1 with $\gamma-2$ or $\gamma-3$, or with $\gamma-4$ or $\gamma-8$, did not differ within each set. There were no significant differences between the TARPs at an interpulse interval of $500 \mathrm{~ms}$.

To further investigate the effect of TARPs on receptor recovery from desensitization, we performed conventional two-pulse protocols (Fig. 2A-E). Briefly, an initial sustained application of $10 \mathrm{~mm}$ glutamate $(50$ or $100 \mathrm{~ms}$ ) was used to establish steadystate desensitization. This application was subsequently repeated at various intervals after completing the first application. The envelope of the peak currents evoked by the second application potentially indicate the rate at which the receptors recover from desensitization. However, changes in other rate constants can also influence the envelope of the peak currents. In order to pool results from multiple patches, the amplitude of the peak current evoked by the second application (measured from the steadystate current at the end of the first response) was expressed as a fraction of the amplitude of the peak current evoked by the first application with which it was paired. Mean results for GluA1 alone and for GluA1 co-expressed with each of the four TARP isoforms are shown in Figure $2 F$. All four TARPs significantly hastened recovery from desensitization. When the data for 

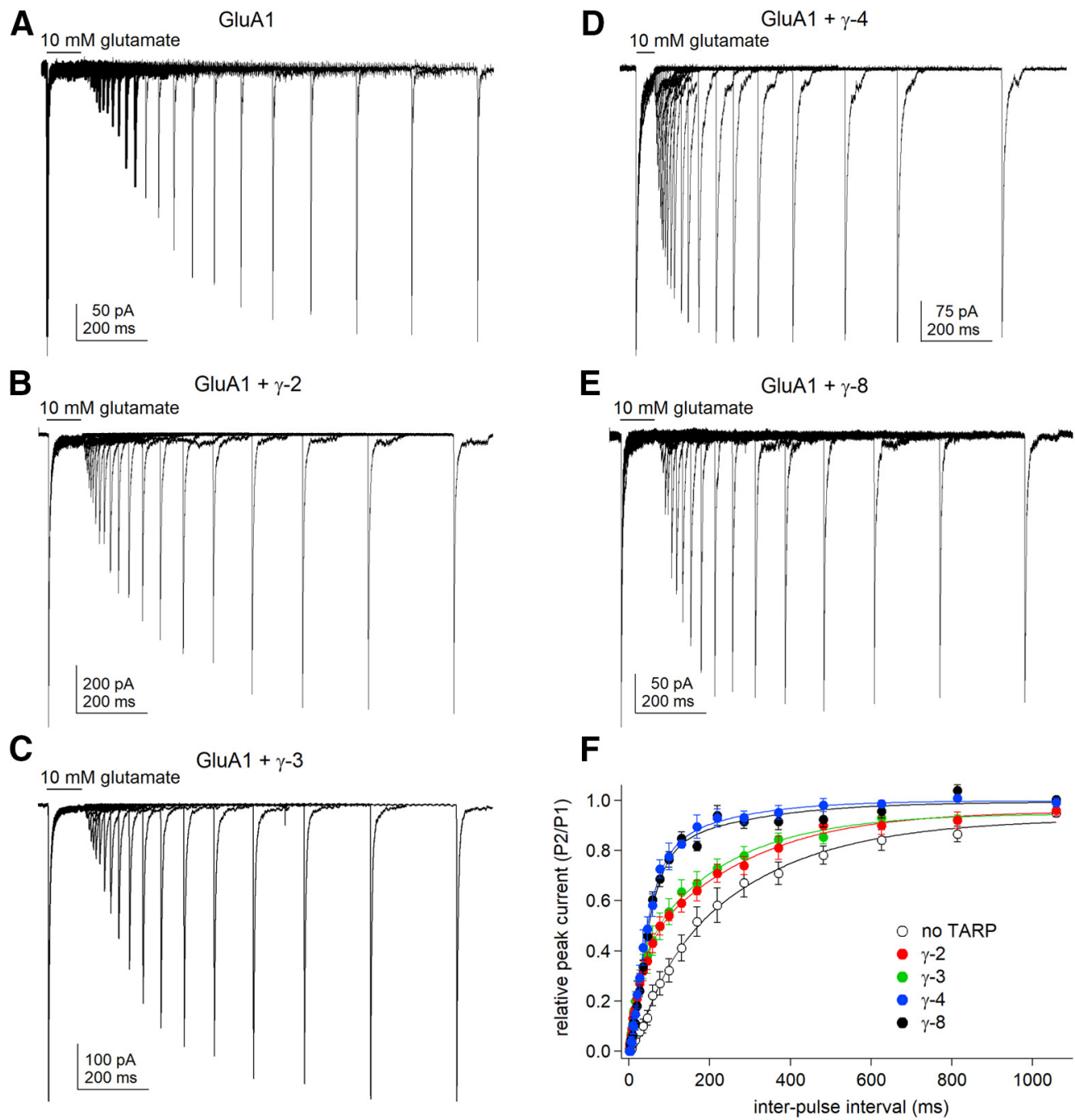

Figure 2. Distinct effects of TARP isoforms on recovery from desensitization. $A-E$, Results obtained by using two-pulse recovery protocols for GluA1 alone (no TARP) and GluA1 co-expressed with the indicated TARPs. Sustained applications of $10 \mathrm{~mm}$ glutamate ( $100 \mathrm{~ms}$ for $\boldsymbol{A}-\boldsymbol{C}, \boldsymbol{E}$, or $50 \mathrm{~ms}$ for $\boldsymbol{D}$; indicated with black bars above traces) were followed by a second application of 10 mм glutamate that was made at increasing interpulse intervals. $\boldsymbol{F}$, Quantification of recovery from desensitization data are presented in panels $\boldsymbol{A}-\boldsymbol{E}$. The results are expressed as the peak amplitude of the second response relative to the first response (P2/P1). The peak amplitudes were measured from the steady-state current at the end of the first application of glutamate. The data (mean \pm SEM) for each group were pooled ( $n=5$ or 6 patches per group). The fitting curves are composed of double H-H-type components.

specific interpulse intervals were compared with one-way ANOVA, the recovery associated with each Type I TARP was found to be greater than the recovery achieved with GluA1 alone at intervals longer than $20 \mathrm{~ms}$ and shorter than $200 \mathrm{~ms}$ $(p<0.05)$. In addition, the recovery time courses for $\gamma-2$ and $\gamma-3$ were nearly identical, while TARPs $\gamma-4$ and $\gamma-8$ similarly mediated significantly faster recovery than $\gamma-2$ or $\gamma-3$ (Fig. 2). These results are consistent with desensitization and deactivation data previously reported for each TARP isoform (Cho et al., 2007). As a result, the four Type I TARPs have been divided into two subgroups based on their speed of receptor recovery from desensitization. However, while several traces of GluA1 with TARPs showed rebounding of current (Fig. 2), rebounding was not consistently observed in this study. For example, a total of $24 \pm 9 \%$ of the traces showed rebounding events, and their incidence was random. The latter result is consistent with modal gating (Zhang et al., 2014) than superactivation, which occurs more consistently with prolonged glutamate application (Carbone and Plested, 2016).

We previously reported that the recovery time course for GluA1 is sigmoidal in shape, and is adequately fitted by a
$\mathrm{H}$-H-type equation. For the latter, the current at time $t$ is $I_{\mathrm{t}}=$ $\left(I_{\max }{ }^{1 / m}-\left(I_{\max }{ }^{1 / m}-I_{0}{ }^{1 / m}\right) \exp (-\mathrm{t} / \tau)\right)^{m}$, where $I_{0}$ is the current at $t=0, I_{\max }$ is the peak current at long interpulse intervals, $\tau$ is the recovery time constant, and the exponent, $m$, represents the number of equivalent independent steps along the recovery pathway. In Figure 2, the fit to the mean data for GluA1 alone produced a time constant of $196 \mathrm{~ms}$ and an $m$ value of 1.4 , consistent with previous results (Robert et al., 2001). When GluA1 receptors were co-expressed with TARPs, the recovery time courses still exhibited a good fit with the double $\mathrm{H}-\mathrm{H}$-type equation, especially for the short interpulse intervals (Fig. 3). The data are also similar to those reported for GluA2 in a previous study (Zhang et al., 2006). The expanded time-scaled insets (Fig. $3 A$ ) illustrate where differences in the double $\mathrm{H}-\mathrm{H}$-type equation fit were observed. This fit estimates both fast $\left(\tau_{1}\right)$ and slow time $\left(\tau_{2}\right)$ constants, as well as the fraction of fast (R1) and slow (R2, $\mathrm{R} 1+\mathrm{R} 2=1)$ components. Weighted $\tau$ recovery $\left(\tau_{\text {recovery }}\right)$ was calculated with the equation: $\mathrm{R} 1{ }^{\star} \tau_{1}+\mathrm{R} 2{ }^{\star} \tau_{2}$. As shown in Figure $2 F$, the four Type I TARPs can be categorized according to weighted $\tau$ values to form two subgroups. While all of the TARP subtypes exhibited reduced weighted $\tau$ values compared 

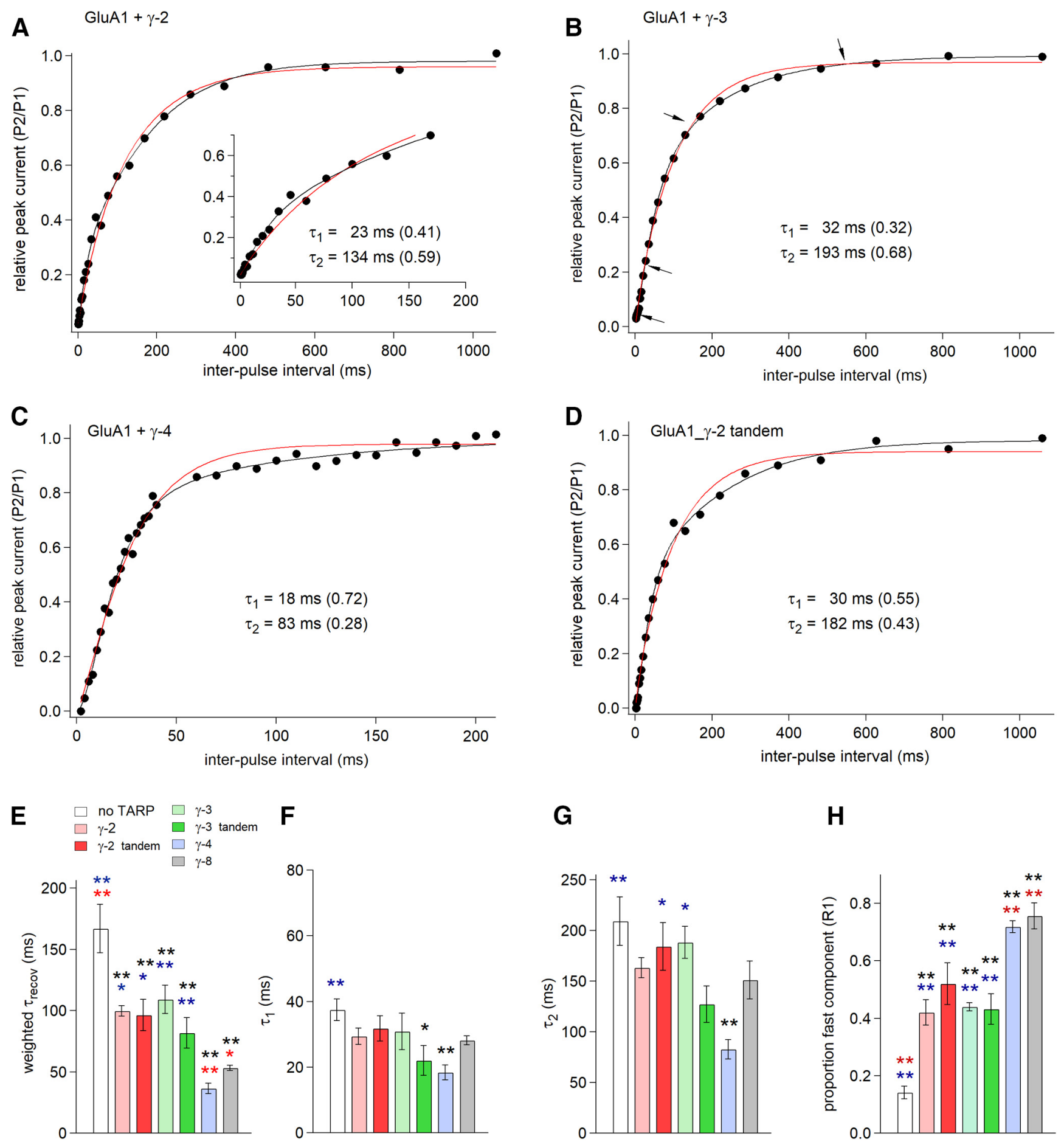

\section{$\mathbf{F}$}

G

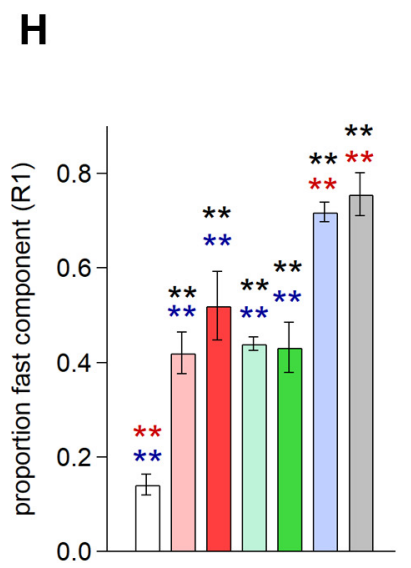

Figure 3. TARP isoforms enhance a fast component of recovery. $\boldsymbol{A}-\boldsymbol{D}$, Recovery data obtained following sustained $100-\mathrm{ms}$ applications of $10 \mathrm{~mm}$ glutamate to individual patches from cells co-transfected with GluA1 and $\gamma-2(\boldsymbol{A}), \gamma-3(\boldsymbol{B})$, or $\gamma-4(\boldsymbol{C})$, or transfected with GluA1_ $\gamma-2$ tandem $(\boldsymbol{D})$. The red and black curves in each panel are one- and two-component H-Htype fits of the results, respectively. The data deviate systematically from the one-component fits (arrows in panel $\boldsymbol{B}$ point to such crossing points). In panel $\boldsymbol{A}$, the time scale of the inset is expanded to better show the results obtained at short interpulse intervals. The time constants (relative amplitudes) from the two component fits are shown. $\boldsymbol{E}-\boldsymbol{H}, \mathbf{Q u a n t i f i c a t i o n}$ of the recovery data for GluA1 alone (without TARP), GluA1 co-expressed with each of the Type I TARPs, and the two GluA1_TARP tandems ( $n=5-7$ patches per group). Data are presented as the mean \pm SEM for the weighted $\tau$ of recovery $(\boldsymbol{E})$, time constants $(\boldsymbol{F}, \boldsymbol{G})$, and the relative amplitude of the fast recovery component $(\boldsymbol{H})$. For each parameter, the values were compared with one-way ANOVA followed post hoc with Bonferroni's multiple comparisons $(\boldsymbol{E}-\boldsymbol{H}) ;{ }^{*} p<0.05$, ${ }^{* *} p<0.01,{ }^{* * *} p<0.001$. Different colored asterisks indicate a significant difference compared with "no TARP" (black), $\gamma-2$ (red), and $\gamma-4$ (blue).

with GluA1 without TARP (no TARP; Fig. 3E), the weighted $\tau$ values for $\gamma-4$ and $\gamma-8$ were significantly smaller than those for $\gamma-2$ and $\gamma-3$ (Fig. 3E). Similarly, all four TARP isoforms significantly increased the proportion of the fast component of recovery compared with the GluA1 receptor (Fig. $3 H$ ), although $\gamma-4$ and $\gamma-8$ significantly increased both the fast and slow time constants of recovery more than $\gamma-2$ and $\gamma-3$ (Fig. $3 F, G$ ). These results suggest that the proportion of each component for each TARP defines two subgroups, $\gamma-2$ and $\gamma-3$ versus $\gamma-4$ and $\gamma-$ 8 , for determining AMPAR recovery kinetics. 
We previously reported that exposure to glutamate causes rapid functional uncoupling of TARP-receptor complexes and a reduction in TARP modulation of receptor gating (MorimotoTomita et al., 2009). Moreover, these effects occur secondary to the conformational changes that accompany glutamate-induced desensitization (Morimoto-Tomita et al., 2009). Therefore, we hypothesized that TARPs mediate distinct effects on recovery from desensitization which are reflected in differences in desensitization-induced functional uncoupling of TARP and receptors. To test this hypothesis, we used tandem constructs with the $\mathrm{C}$ terminus of GluA1 fused directly to the $\mathrm{N}$ terminus of either $\gamma-$ 2 or $\gamma-3$. Previous studies of a GluA1- $\gamma-2$ tandem construct showed that the resulting receptors exhibited many of the signature features of receptor gating modulated by $\gamma-2$ (MorimotoTomita et al., 2009). For example, the GluA1- $\gamma-2$ tandem slowed desensitization and enhanced steady-states to a similar extent as GluA1 co-expressed with $\gamma-2$. In Figure $3 D-H$, recovery from desensitization for the two GluA1 tandem constructs tested in the present study is shown. Both tandems enhanced recovery from desensitization similar to co-expression of GluA1 with $\gamma-2$ and $\gamma-3$, and the results from each tandem did not differ from each other (Fig. 3E-H).

\section{TARPs speed recovery from desensitization more effectively for brief applications of glutamate than for sustained applications of glutamate}

Presynaptic spike rate and release probability are two factors which primarily determine the frequency of fast synaptic transmission. As an important property of AMPAR, recovery from desensitization also contributes to the frequency of fast synaptic transmission during repeated glutamate release from presynaptic terminals (Trussell and Fischbach, 1989; Trussell et al., 1993; Chen et al., 2002; DiGregorio et al., 2007; Devi et al., 2016; Lu et al., 2017). It has been demonstrated that fast synaptic transmission can be mimicked with brief applications of glutamate to receptors. In a previous study, both $\gamma-2$ and $\gamma-8$ were able to reduce paired-pulse depression in response to brief applications of glutamate (Cho et al., 2007). In the present study, all four TARPs were able to speed recovery from desensitization induced by sustained glutamate applications (Figs. 2, 3). In contrast, brief applications of glutamate did not achieve complete desensitization of all channels. Therefore, we compared the effect of $\gamma-2$ and $\gamma-3$ on GluA1 recovery following both brief and sustained applications of glutamate. Both $\gamma-2$ and $\gamma-3$ were able to speed recovery following a brief application of glutamate more effectively than following a sustained application of glutamate (Fig. 4). When recovery of GluA1 with $\gamma-2$ was compared between the two application protocols, the receptors appeared to begin recovery following a brief application of glutamate, while the major proportion of receptors remained in a desensitized state following a sustained application of glutamate (Fig. 4A, right panel). To directly compare recovery from brief and sustained applications of glutamate, we measured both recoveries from the same patch of GluA1 with $\gamma-3$. Faster receptor recovery was observed following brief applications of glutamate rather than following sustained applications when different time points were compared (Fig. $4 B$ ). Each plot of mean $\gamma-2$ and $\gamma-3$ data fit well with the double $\mathrm{H}$-H-type equation (Fig. $4 C$ ). In addition, when recovery time courses from these two protocols were fitted with the double $\mathrm{H}-\mathrm{H}$-type equation, the time constants for both the slow and fast components were significantly faster for the brief applications of glutamate than for the sustained applications of glutamate (Fig. 4D). However, the proportion of the fast component of recovery (R1) did not statistically differ between the brief and sustained applications of glutamate (Fig. 4D). The mean data obtained for $\gamma-2$ and $\gamma-3$ also did not significantly differ ( $p>0.05$; two-tailed Student's $t$ test). Similarly, the mean plot of $\gamma-2$ and $\gamma-3$ pooled data showed faster recovery from brief glutamate applications than from sustained applications of glutamate (Fig. $4 E$ ). Taken together, these results suggest that TARP speeds the release of a large number of channels from desensitization states during a brief application of glutamate. Furthermore, the proportion of fast component which escapes desensitization and is available to open and respond to the next glutamate pulse appears to be TARP isoform dependent, yet is independent of the initial duration of glutamate, despite the speed of recovery being faster with a brief pulse.

To validate the above hypothesis, we tested varying durations of glutamate applications on receptors co-expressed with $\gamma-2$ and $\gamma-3$ (Fig. 5). Our results demonstrate that the longer the receptors are subjected to glutamate, the higher the proportion of receptors that are in a desensitized state at the end of the application. In addition, recovery from desensitization appears to be influenced by the duration of the first application of glutamate. For example, for the same interpulse interval, the recovery proportion was much larger for a 2-ms preapplication than for a 10ms application (Fig. 5A, $B$, pink and blue lines, respectively). The largest proportion was observed at 10 - and 20-ms interpulse intervals (the time intervals between glutamate-off timing of the first pulse and glutamate-on timing of the second pulse) for receptors expressed with $\gamma-2$ and $\gamma-3$ (Fig. 5A,B). However, when the duration of the first pulse was longer than $20 \mathrm{~ms}$, the proportion of channel recovery was similar (Fig. 5C). These results indicate that most of the channels entered a desensitization state, and then a similar recovery from desensitization was observed when the duration of the first pulse was $50 \mathrm{~ms}, 100 \mathrm{~ms}$, or longer (Fig. 5C). When the paired-pulse ratio with different glutamate application times for the first pulse was fitted by an exponential function, the time constants $\left(\tau_{\mathrm{P} 1}\right)$ were $\sim 4 \mathrm{~ms}$ for $\gamma-2$ and $\gamma-3$ at the $10-\mathrm{ms}$ interpulse interval (Fig. 5C,D). These results indicate that the shorter the duration of the first glutamate application, the greater the number of receptors which will respond to glutamate re-stimulation at the same interpulse interval (Fig. 5C). At 10-ms interpulse intervals, only $10 \%$ of the receptors recovered from desensitization when the first application time exceeded $20 \mathrm{~ms}$ (Fig. $5 C$ ). Time constants $\left(\tau_{\mathrm{P} 1}\right)$ were also found to be independent of the interpulse interval (Fig. 5D). Taken together, these data indicate that the proportion of channels entering different desensitization states is independent of the duration of glutamate exposure.

\section{Role of the Ex1 domain in TARP isoform-specific modulation of recovery}

Previous studies of chimeric receptors have shown that TARP modulation of activation gating is largely determined by sequence elements present within their first extracellular domain (Ex1; (Tomita et al., 2005; Cho et al., 2007; Milstein et al., 2007). Given the above-noted differences between the two TARP subfamilies in modulating desensitization and deactivation gating, our recovery data further suggest that the two TARP subfamilies $(\gamma-$ $2, \gamma-3$ and $\gamma-4, \gamma-8)$ also differ with respect to TARP modulation of recovery from desensitization (Figs. 2, 3). To test whether isoform-specific differences in modulation of recovery also depend on sequence elements within Ex1, we compared recovery when GluA1 was co-expressed with $\gamma-2$ or $\gamma-4$, and when the 
A
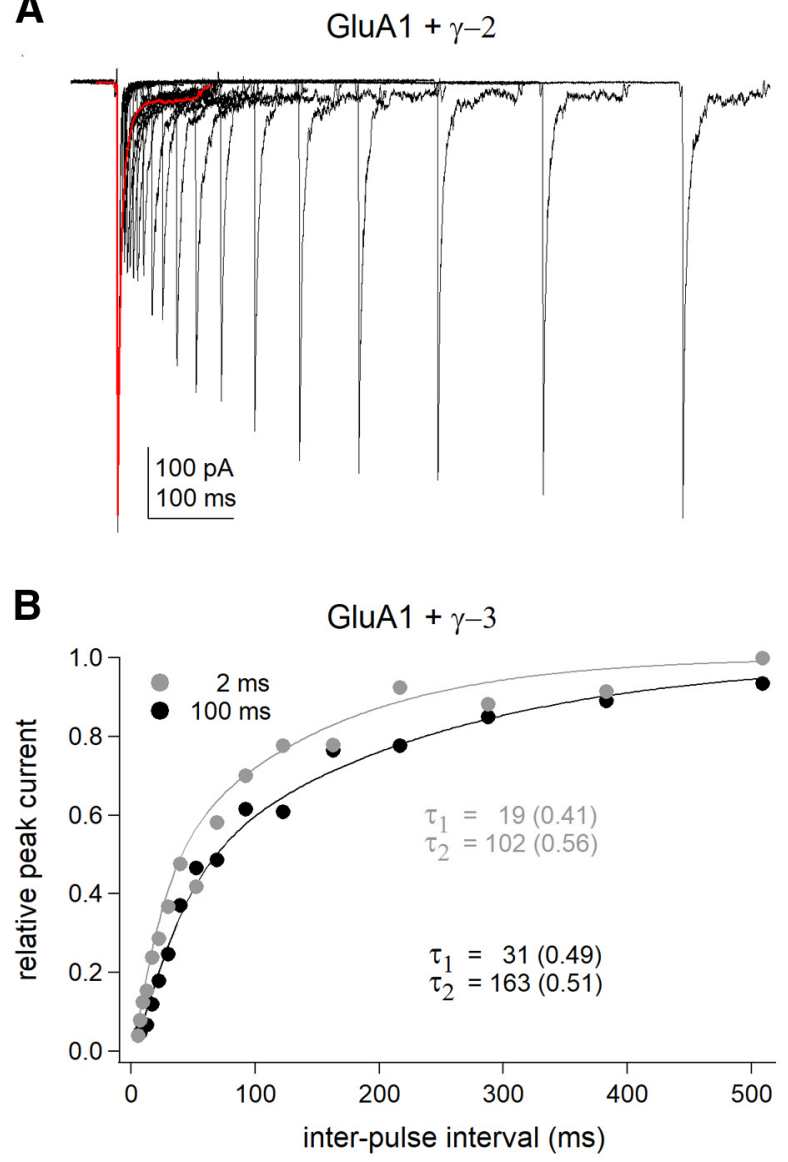

D

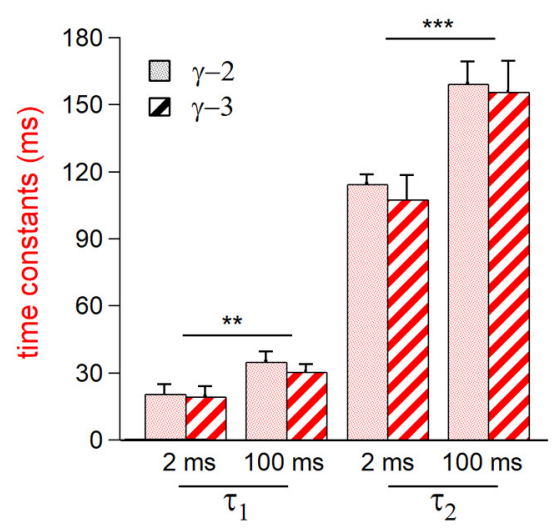

$10 \mathrm{mM}$ glutamate

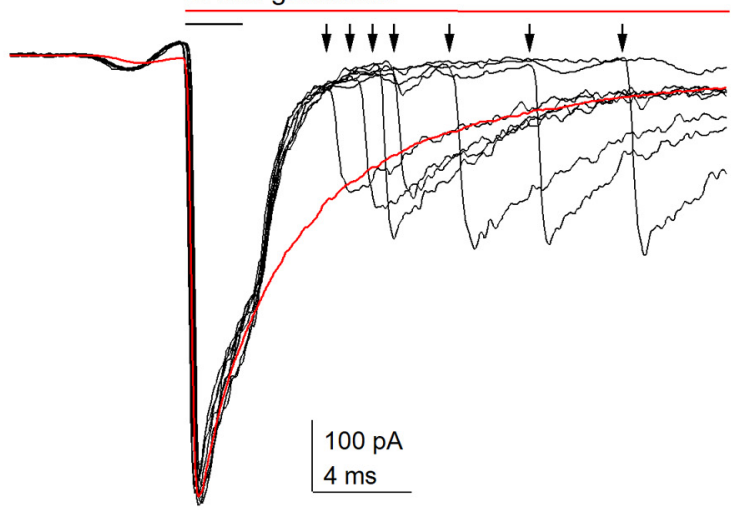

C

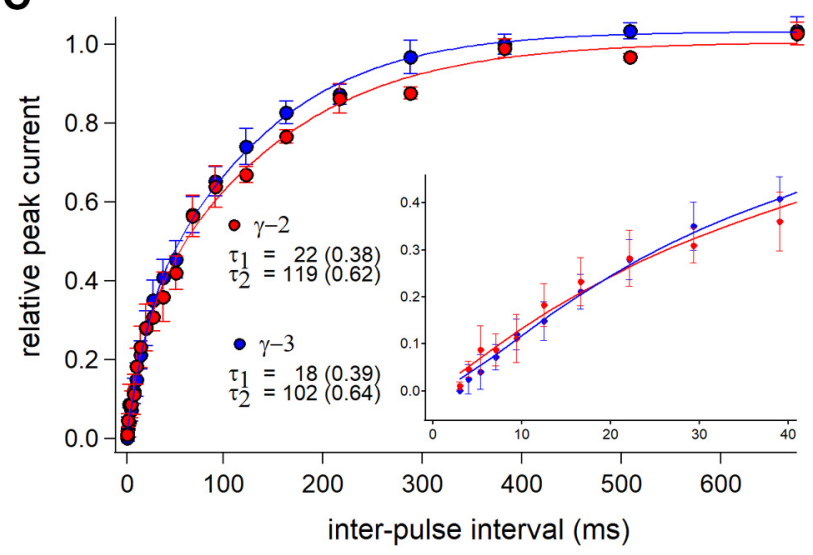

$\mathbf{E}$

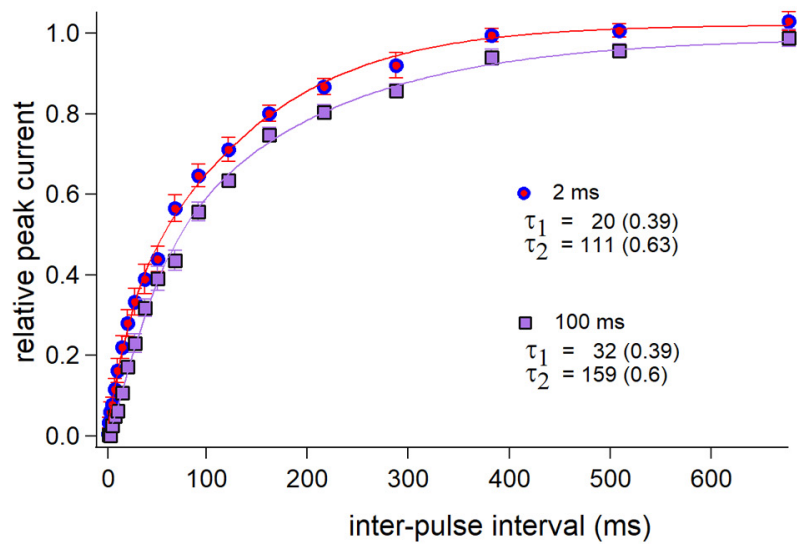

Figure 4. Duration of glutamate application contributes to the time constants of recovery. A, Data obtained from a two-pulse recovery protocol (black traces) with 2- and 100-ms applications of $10 \mathrm{~mm}$ glutamate (indicated with black bars above traces on the right), respectively. The patch was excised from a cell co-transfected with GluA1 and $\gamma-2$. The left panel shows the complete dataset, while the right panel shows the results for the first seven interpulse intervals on an expanded time scale (arrows indicate the onset of the second glutamate application). In each panel, the red traces show the inward current (average of 20 responses) elicited by a single 100-ms application of $10 \mathrm{~mm}$ glutamate (indicated with red bars above the traces on the right) to the same patch immediately before the recovery protocol was run. $\boldsymbol{B}, \mathrm{A}$ more rapid recovery is observed when the first glutamate application is short. Recovery data were obtained from the same patch co-expressing GluA1 with $\gamma-3$ from protocols in which the first application of glutamate was either $2 \mathrm{~ms}$ (gray circles) or $100 \mathrm{~ms}$ (black circles) in duration. For interpulse intervals shorter than $100 \mathrm{~ms}$, the peak amplitude of the second response was estimated from the amplitude evoked by a 100 -ms application at the same postapplication time. For intervals of $100 \mathrm{~ms}$ or more, the peak amplitude of the second response was measured from the amplitude of the steady-state current. C, The mean plot for $\gamma-2$ and $\gamma-3$ for the first 2-ms application of glutamate is provided in the inset with an expanded initial time scale. The curve fits to each set of results contain two $\mathrm{H}-\mathrm{H}$ components. Values from the fit are provided under their respective trace symbols. D, Quantification of recovery data from GluA1 with either $\gamma-2$ or $\gamma-3$ as the time constants $\left(\tau_{1}, \tau_{2}\right)$ of the fast and slow components, and the relative proportion of the fast component (R1). Comparison of data for the brief 2-ms ( $n=4$ for $\gamma-2$ and $n=6$ for $\gamma-3$ ) and 100-ms sustained $(n=9$ for $\gamma-2$ and $n=6$ for $\gamma-3)$ first applications of glutamate to GluA1 with either $\gamma-2$ or $\gamma-3$. The time constant for each component is faster when the first application is short. However, the proportion of the fast component (R1) did not vary with the duration of the first application. $\boldsymbol{E}$, The mean plot of recovery following brief and sustained applications of glutamate of pooled data from $\gamma-2$ and $\gamma-3$ ( $n=10-15)$. Since the mean parameters were similar between $\gamma-2$ and $\gamma-3$, both sets of data were pooled for comparison of brief and sustained pulses of initial glutamate application. Data are presented as mean \pm SEM; ${ }^{* *} p<0.01,{ }^{* * *} p 0.001$, two-tailed Student's $t$ test. 
GluA1 $+\gamma-2$

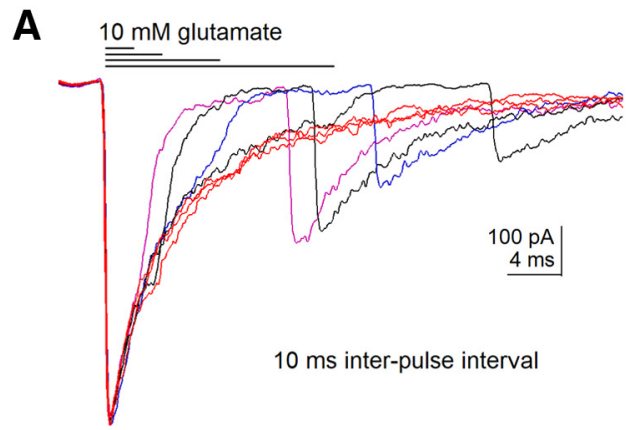

\section{C}

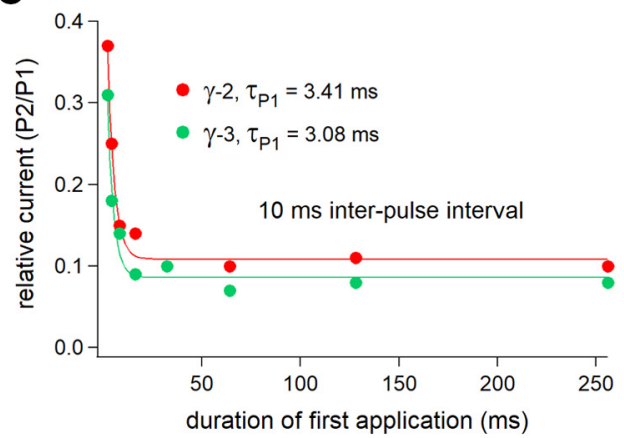

GluA1 $+\gamma-3$

B

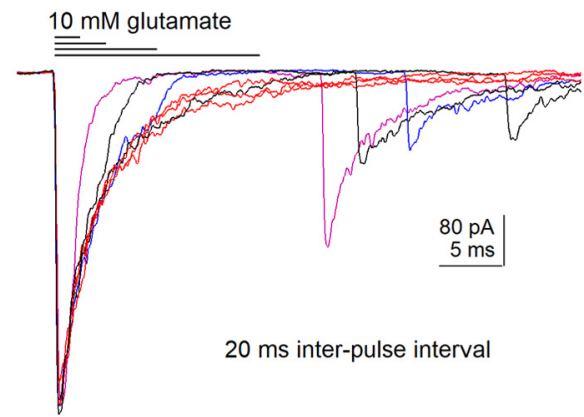

D

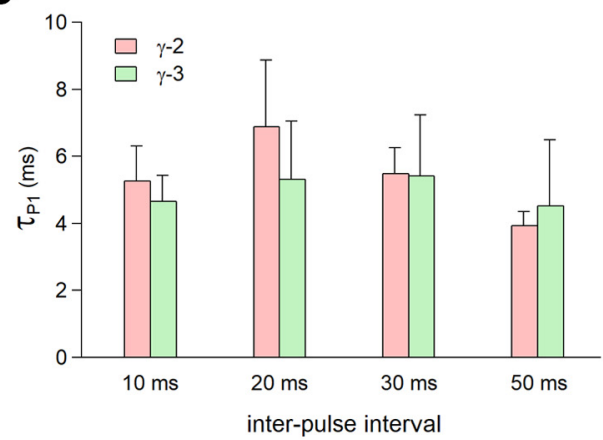

Figure 5. Recovery from desensitization depends on the duration of glutamate exposure, but not on the interpulse interval. $\boldsymbol{A}, \boldsymbol{B}$, Representative responses of GluA1 co-expressed with either $\gamma-2$ ( $\boldsymbol{A}, 10$-ms interpulse interval) or $\gamma-3$ (B, 20-ms interpulse interval) to paired-pulse (each trace shown in different colors) or sustained (red traces) glutamate applications. Paired-pulse protocols varied the duration of the first application of $10 \mathrm{~mm}$ glutamate while keeping the interval between the first and second applications constant. The peak amplitude of the second response declined as the duration of the first application increased. C, Quantification of a 10-ms interpulse interval trace for $\gamma-2$ and $\gamma-3$ shows relative current (ratio of the second and first peak amplitudes) plotted as a function of the duration of the first application. The peak amplitude of the second response was estimated by subtracting the current evoked by a 100-ms application of glutamate as the baseline (similar to the red traces in $\boldsymbol{A}, \boldsymbol{B})$. The results were fitted with exponential functions to determine the indicated time constants $\left(\tau_{\mathrm{P} 1}\right)$. $\boldsymbol{D}$, Time constants $\left(\tau_{\mathrm{P} 1}\right)$ obtained for $\gamma-2$ and $\gamma-3$ (which did not significantly differ) at four different interpulse intervals ( $n=3$ or 4 values per group). Data are presented as mean \pm SEM.

Ex1 domains of TARPs $\gamma-2$ and $\gamma-4$ were interchanged in two chimera constructs.

Swapping the Ex1 domain of $\gamma-4$ with that of $\gamma-2$ to form $\gamma-4(\gamma-2 \mathrm{Ex} 1)$ resulted in slower recovery from desensitization and a recovery time course similar to that for $\gamma-2$ (Fig. 6). Similarly, replacement of the Ex 1 domain of $\gamma-2$ with the Ex1 domain from $\gamma-4$ to create $\gamma-2(\gamma-4 \mathrm{Ex} 1)$ also slowed recovery from desensitization (rather than speeding it; Fig. $6 A, B$ ). However, $\gamma-2(\gamma-4 \mathrm{Ex} 1)$ exhibited a recovery time course which differed from those of native $\gamma-4$ and $\gamma-2$ (Fig. 6A,B). These results indicate that glutamate receptor recovery is not only modulated by the Ex1 domain, but also by other regions of TARPs. This is an unexpected result since the two chimeras studied, $\gamma-2(\gamma-4 \mathrm{Ex} 1)$ and $\gamma-4(\gamma-2 \mathrm{Ex} 1)$, were predicted to show inverse differences in modulation of desensitization gating based on our previous characterization of $\gamma-2$ and $\gamma-4$ (Cho et al., 2007).

\section{Both Ex1 and Ex2 domains contribute to TARP isoform- specific modulation of recovery}

TARPs are transmembrane proteins which contain two extracellular domains (Ex1 and Ex2; Tomita et al., 2005). Both Ex1 and Ex2 of $\gamma-2$ have been shown to contribute to regulation of AMPA receptor gating (Twomey et al., 2016; Zhao et al., 2016; Ben-Yaacov et al., 2017; Riva et al., 2017). The results presented above indicate that speeding of recovery by TARPs is not determined by the Exl domain alone. To further investigate the observed difference in recovery for the two kinds of TARPs, two additional chimera constructs were assembled with the extracellular domains of $\gamma-2$ and $\gamma-4$ swapped. These double chimeras were co-expressed with GluA1 to compare recovery from desensitization. The $\gamma-2$ double chimera with the Ex1 and Ex2 domains of $\gamma-4[\gamma-2(\gamma-4 \operatorname{Ex} 1 \mathrm{Ex} 2)]$ did speed recovery from desensitization. Moreover, the recovery time course was similar to that of $\gamma-4$. When the Ex1 and Ex2 domains of $\gamma-4$ were replaced with the Ex1 and Ex2 domains of $\gamma-2[\gamma-4(\gamma-$ 2Ex1Ex2)], recovery from desensitization was also slowed, and the recovery time course was similar to that observed with coexpression of $\gamma-2$ (Fig. $6 C-F$ ).

As described above, a brief application of glutamate was found to speed recovery from desensitization for receptors coexpressed with $\gamma-2$ and $\gamma-3$ (Fig. 4). Therefore, we wanted to investigate whether the $\gamma-2(\gamma-4 \operatorname{Ex} 1 \mathrm{Ex} 2)$ and $\gamma-4(\gamma-2 \mathrm{Ex} 1 \mathrm{Ex} 2)$ double chimeras would also exhibit the same characteristic. Following expression of GluA1 and $\gamma-4(\gamma-2 \mathrm{Ex} 1 \mathrm{Ex} 2), \sim 80 \%$ of the receptors recovered from desensitization after a short pulse of glutamate (Fig. $7 A-C$ ), whereas only $\sim 50 \%$ of the receptors recovered following a sustained pulse of glutamate (Figs. $7 C$, dashed line, $6 E$ ) at the 100-ms interpulse interval. Similar to $\gamma-2$ and $\gamma-3$ (Fig. $4 C$ ), the proportion of the fast recovery component was similar for both chimeras with brief and sustained applications of glutamate. However, for $\gamma-2(\gamma-4 \mathrm{Ex} 1 \mathrm{Ex} 2)$, the proportion of the fast component of recovery for both the brief and sustained pulses reached $\sim 80 \%$ (similar to $\gamma-4)$, while the proportions for $\gamma-4(\gamma-$ 2Ex1Ex2) were $\sim 40 \%$ (similar to $\gamma-2$; Fig. $7 D$ ). Thus, exchanging both the Ex1 and Ex2 domains swapped the recovery phenotypes of $\gamma-2$ and $\gamma-4$. 
A

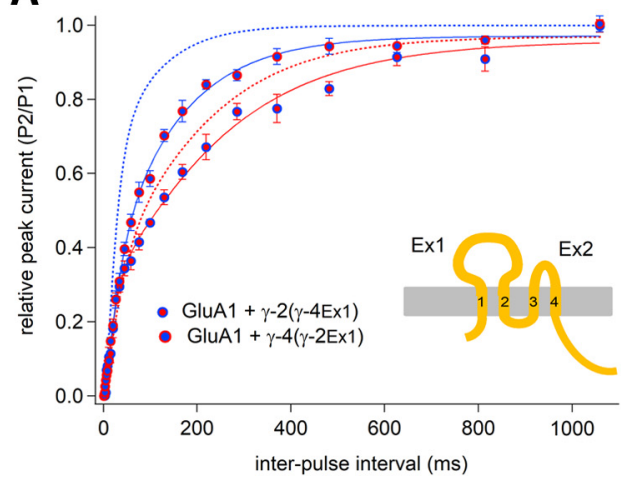

C

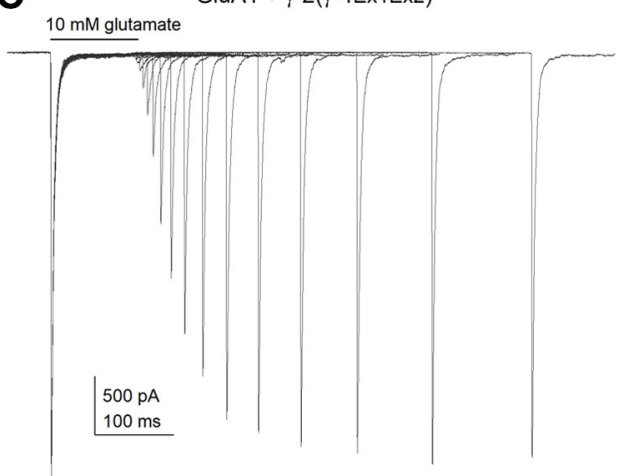

E

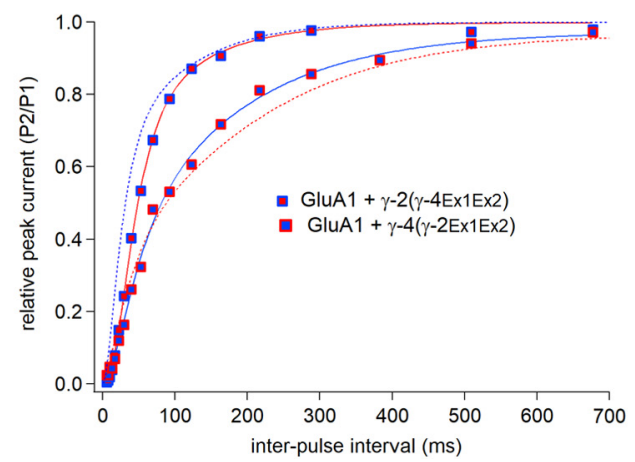

B

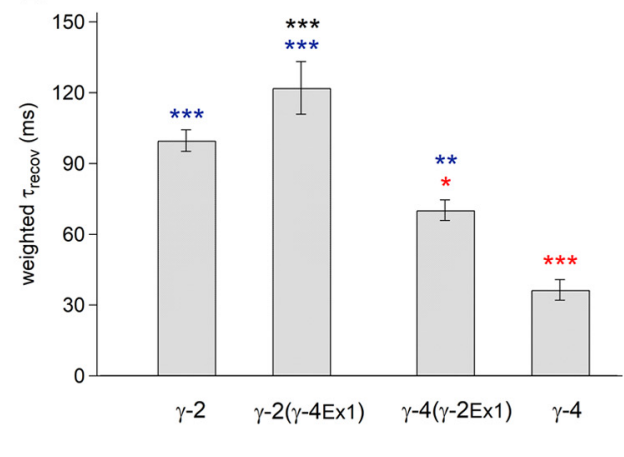

D

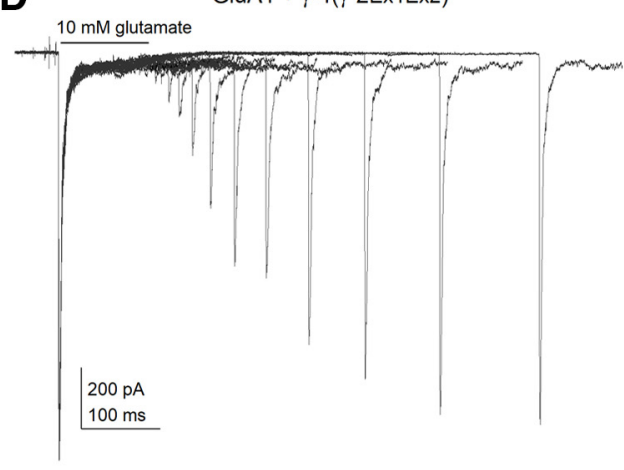

$\mathbf{F}$

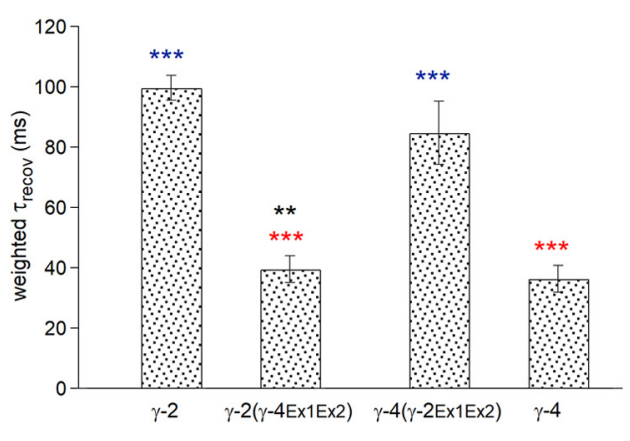

Figure 6. TARP extracellular loops 1 and 2 dictate isoform-specific speeding of recovery from desensitization. $A, B$, Recovery from desensitization for GluA1 with TARP $\gamma-2, \gamma-4$, and their chimeras. TARP chimeras were constructed by swapping the first extracellular loop (Ex1) of $\gamma-2$ and $\gamma-4$. The chimeras were then co-expressed with GluA1 and recovery from desensitization was measured from the start of a 100-ms preapplication of $10 \mathrm{~mm}$ glutamate. The red and blue smooth solid curves are the two-component fits to the data for GluA1 with the $\gamma-2$ carrying $\gamma-4$ Ex1 chimera (red circles, blue border, $n=5$ ) and for GluA1 with the $\gamma-4$ carrying $\gamma-2$ Ex1 chimera (blue circles, red border, $n=7$ ), respectively. Inserting the Ex1 domain of $\gamma-2$ into $\gamma-4$ slows recovery and produces a recovery time course which is more similar to $\gamma-2$. Inserting the $\gamma-4$ Ex 1 domain into $\gamma-2$ slows recovery as well. GluA1 with TARP $\gamma-4$ (blue dotted curves) and GluA1 with TARP $\gamma-2$ (red dotted curves) from Figure 2 are shown. B, Quantification of the weighted time constant of recovery $\left(\tau_{\text {recov }}\right)$ for $\gamma-2, \gamma-4$, and the two Ex1 chimeras. $\boldsymbol{C}, \boldsymbol{D}$, Representative two-pulse recovery protocols performed on patches excised from cells co-expressing GluA1 and TARP chimeras in which both extracellular domains (Ex1 and Ex2) were swapped between $\gamma-2$ and $\gamma-4$. E, Recovery data in $\boldsymbol{C}, \boldsymbol{D}$ were fitted with two-component H-H-type equations. (0-expression of the $\gamma-2(\gamma-4$ Ex1Ex2) chimera results in faster recovery than co-expression of the $\gamma-4$ chimera containing the two extracellular domains of $\gamma-2 . \gamma-4$ (blue dotted curves), $\gamma-2$ (red dotted curves). Comparison data are from Figure 2. $\boldsymbol{F}$, Summary of weighted recovery time constants for GluA1 receptors co-expressed with $\gamma-2, \gamma-4$, or one of the two TARP double chimeras ( $n=5-8$ values per group). Data are presented as mean $\pm \mathrm{SEM} ;{ }^{*} p<0.05,{ }^{* *} p<0.01$, ${ }^{* * *} p<0.001$, one-way ANOVA followed post hoc by Bonferroni's multiple comparisons. Different colored asterisks indicate a significant difference compared with $\gamma-2$ (red), $\gamma-4$ (blue), and $\gamma-4(\gamma-2$ Ex1) in $\boldsymbol{B}$ or $\gamma-4(\gamma-2$ Ex1Ex2) in $\boldsymbol{F}$ (black).

\section{The Ex2 domain determines the proportion of different components of recovery}

We observed that the recovery time courses of the double chimeras, $\gamma-2(\gamma-4 \operatorname{Ex} 1 \mathrm{Ex} 2)$ and $\gamma-4(\gamma-2 \operatorname{Ex} 1 \mathrm{Ex} 2)$, closely approximate those of $\gamma-4$ and $\gamma-2$, respectively. In contrast, an exchange of the Ex1 domain alone did not produce a similar effect. To test the hypothesis that the Ex2 domain is responsible for modulating AMPA receptor recovery from desensitization, the Ex2 domain was exchanged between $\gamma-2$ and $\gamma-4$, and then recovery from desensitization was examined for each chimera. Recovery data following a sustained pulse of glutamate is shown in Figure 8 for $\gamma-2(\gamma-4 \operatorname{Ex} 2)$ and $\gamma-4(\gamma-2 \mathrm{Ex} 2)$. Indeed, replacing the Ex2 domain of $\gamma-2$ with that of $\gamma-4$ to form $\gamma-2(\gamma-$ $4 \mathrm{Ex} 2$ ) did speed recovery from desensitization (Table 1; Fig. 8A, 

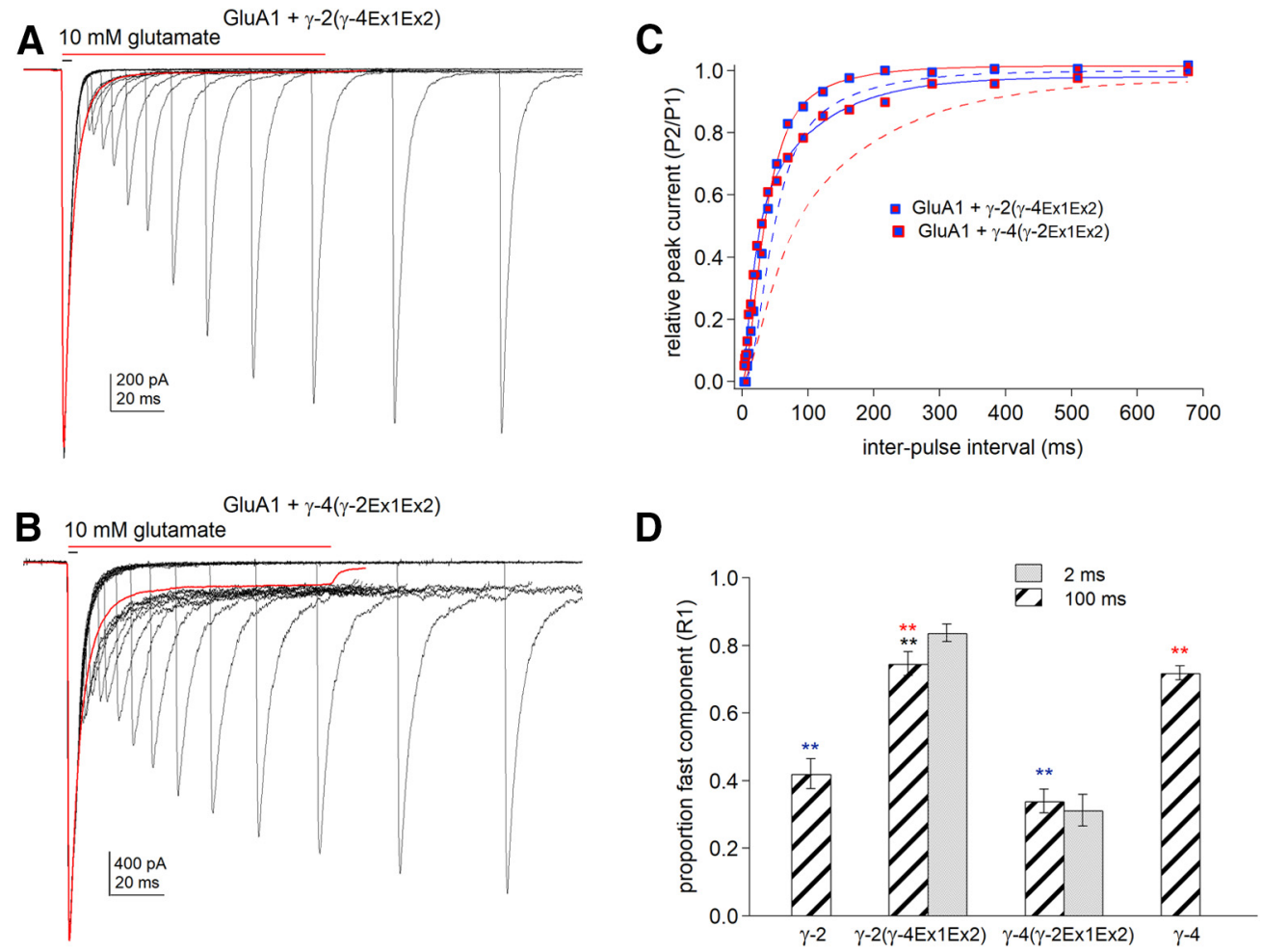

Figure 7. The effect of TARP extracellular domains on recovery from brief glutamate exposure. Responses of patches co-transfected with GluA1 and TARP chimeras to brief ( $2 \mathrm{~ms})$ and sustained (100 ms) glutamate applications were measured. $\boldsymbol{A}, \boldsymbol{B}$, Two-pulse recovery protocols for the TARP double Ex chimeras $[\gamma-2(\gamma-4$ Ex1Ex2) and $\gamma-4(\gamma-2$ Ex1Ex2)] to a 2-ms application of $10 \mathrm{~mm}$ glutamate. Red traces represent the current evoked in the respective patches by a $100-\mathrm{ms}$ application of $10 \mathrm{~mm}$ glutamate just before collecting the recovery data. An average of 20 responses were used as the baseline current for measuring peak current amplitudes evoked by brief pulses at short interpulse intervals. $\boldsymbol{C}$, Recovery data from the traces in $\boldsymbol{A}, \boldsymbol{B}$ fitted with two-component H-H equations (shown as curves). Recovery data in response to 2-ms applications of glutamate are represented by solid lines. Recovery data in response to 100-ms applications of glutamate (shown as dashed lines) were taken from Figure 6 for comparison. $\boldsymbol{D}$, Quantification of the proportion of the fast component of recovery (R1) obtained from fitting recovery data from protocols with brief $(2 \mathrm{~ms})$ and sustained $(100 \mathrm{~ms})$ first applications of glutamate for the two TARP chimeras and the corresponding values from sustained first application protocols for wild-type $\gamma-2$ and $\gamma-4(n=3-8)$. Data are presented as mean $\pm \mathrm{SEM} ;{ }^{* *} p<0.01$, one-way ANOVA followed post hoc by Bonferroni's multiple comparisons. Different colored asterisks indicate a significant difference compared with $\gamma-2$ (red), $\gamma-4$ (blue), and $\gamma-4(\gamma-2$ Ex1Ex2) (black).

C). Furthermore, the recovery time course was much faster than that observed with $\gamma-2$. Conversely, the chimera with the Ex2 domain of $\gamma-2$ slowed the recovery from desensitization, with a recovery time course similar to $\gamma-2$ (Table 1 ; Fig. $8 B, C$ ). While the time constants of recovery (weighted $\tau$; Table 1) were not fully swapped, the proportion of fast component of recovery (R1) for the Ex2 domain exchange approximated that of $\gamma-2$ and $\gamma-4$. In particular, the R1 value of the $\gamma-4(\gamma-2 \mathrm{Ex} 2)$ chimera was nearly the same as $\gamma-2$ (Table 1 ; Fig. $8 C, D$ ). These results suggest that the Ex 2 domain determines the proportion of fast and slow components.

\section{Kinetic model for GluA1 alone and GluA1 with TARPs}

When the onset of desensitization was analyzed (Table 2), the slow phase of the onset of desensitization, as well as recovery from desensitization, were both observed to correlate well, except for the chimeric mutant, $\gamma-2(\gamma-4 \mathrm{Ex} 1)$. The latter had a Pearson coefficient value of -0.83 , which suggests that its slow phase of desensitization relates to its recovery kinetics. For the 100-ms pulses, the slow phase of desensitization varied between 7.3 and $18 \mathrm{~ms}$ (Table 2), while the slow rate of recovery varied between 80 and $200 \mathrm{~ms}$ (Fig. 3G). Since these two aspects significantly differ, we conclude that the slow phase of desensitization does not correspond to a slow rate of recovery.

Next, we aimed to establish kinetic models for GluA1 alone and GluA1 with TARPs by modifying our previous model to include 16 states (Fig. 9A; 2003 Howe model; Robert and Howe,
2003). When we modeled the recovery of GluA1 alone with 2and 100-ms applications of glutamate in the 2003 Howe model, a faster recovery was predicted for the shorter glutamate application (Fig. 9B). However, when the curves were compared with the experimental values obtained (Fig. 9B), there were differences. To address these differences, we modified the rate constants of the 2003 Howe model. The rate constants in the 2003 Howe model were originally estimated from recovery data fitted with a single H-H-type equation (Robert and Howe, 2003). However, our recovery kinetics fit well with double $\mathrm{H}-\mathrm{H}$-type equations (Figs. 3, 4). In addition, we compared both desensitization and deactivation kinetics. Thus, we modified the rate constants (delta1, from 1800 to 2500; delta2 from 200 to 50; K-2 from 0.41 to 1.5 ) to better fit the double H-H-type equations (Table 3 ), and this is referred to as the 2020 Howe model (M2020). With this revised model, we were able to better simulate GluA1 kinetics (Fig. 9C).

We next modeled TARPed AMPARs. We observed that TARP $\gamma-2$ hastened recovery from both brief and sustained pulses of glutamate (Figs. 2-4). To determine which rate constants can reduce both recovery time constants, we altered each of the rate constants in the 2003 Howe model to represent 10fold increases and 10-fold decreases as extreme cases (Table 4). These systematic changes were used to examine possible effects of the values on the kinetics of desensitization, recovery, and deactivation. Only an increase in gammal reduced substantially recovery following the $100-\mathrm{ms}$ pulse. Therefore, we predict that 

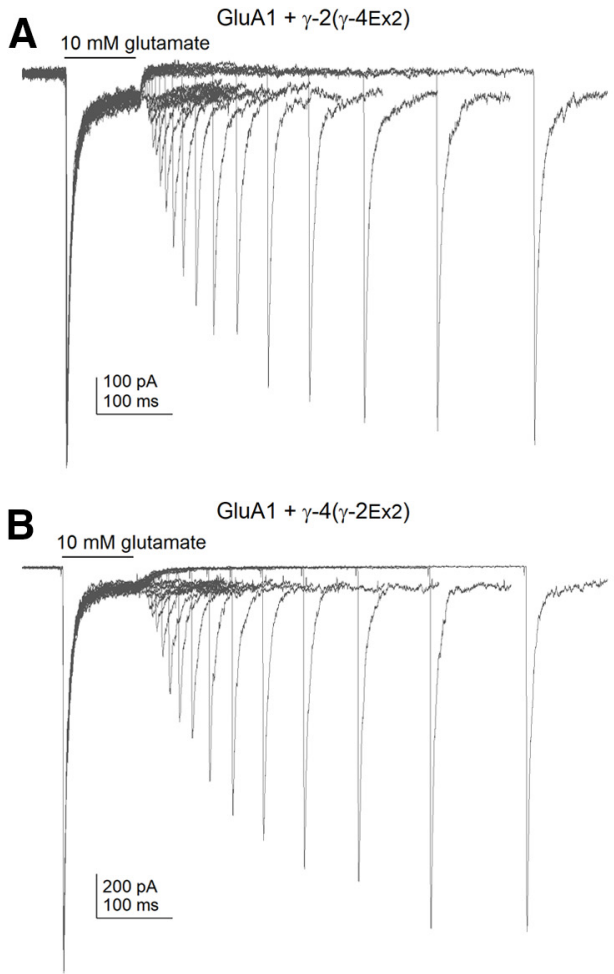

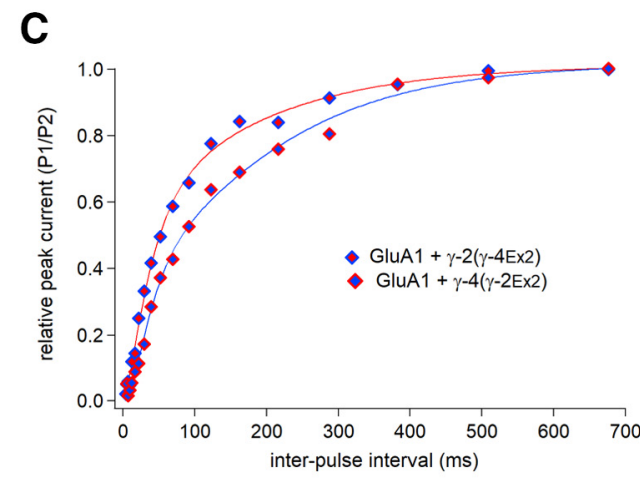

D

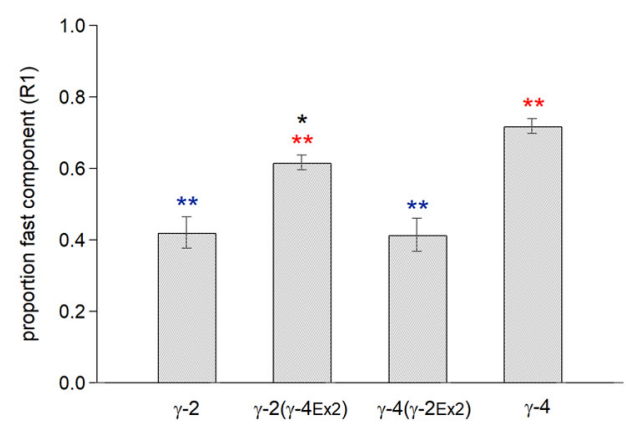

Figure 8. The effect of the Ex2 domain on TARP speeding of recovery from desensitization. TARP chimeras were coexpressed with GluA1 and recovery from desensitization was measured from the start of a 100-ms preapplication of $10 \mathrm{~mm}$ glutamate. $\boldsymbol{A}, \boldsymbol{B}$, Two-pulse recovery protocols for $\gamma-2 / \gamma-4$ chimeras in which the Ex2 domain was swapped. $\boldsymbol{C}$, Recovery data from the protocols in $\boldsymbol{A}, \boldsymbol{B}$ which were fitted (curves) with two-component H-H-type equations. $\boldsymbol{D}$, Bar graphs of mean ( \pm SEM) values for the proportion of the fast component of recovery (R1) obtained for wild-type $\gamma-2$ and $\gamma-4$ compared with the corresponding values for the two Ex2 chimeras. Data are presented as mean \pm SEM; ** $<<0.01$, one-way ANOVA followed post hoc by Bonferroni's multiple comparisons. Different colored asterisks indicate significant differences compared with $\gamma-2$ (red), $\gamma-4$ (blue), and $\gamma-4(\gamma-2$ Ex2) (black).

Table 1. Time constants of recovery from desensitization (mean \pm SEM) for GluA1 co-expressed with various TARPs

\begin{tabular}{lcl}
\hline & Weighted $\tau$ (ms) & Fast component (\%) \\
\hline $\mathrm{A} 1$ & $166.8 \pm 19.9$ & $14.2 \pm 2.2$ \\
$\mathrm{~A} 1+\gamma-2$ & $99.7 \pm 4.3$ & $42.3 \pm 4.4$ \\
$\mathrm{~A} 1+\gamma-3$ & $108.7 \pm 11.7$ & $42.9 \pm 1.4$ \\
$\mathrm{~A} 1+\gamma-4$ & $36.4 \pm 4.4$ & $71.9 \pm 2.1$ \\
$\mathrm{~A} 1+\gamma-8$ & $53.3 \pm 2.3$ & $75.6 \pm 4.6$ \\
$\mathrm{~A} 1 \_\gamma-2$ tandem & $96.5 \pm 12.9$ & $52.0 \pm 7.3$ \\
$\mathrm{~A} 1 \_\gamma-3$ tandem & $81.9 \pm 12.6$ & $43.2 \pm 5.3$ \\
$\mathrm{~A} 1+\gamma-2(\gamma-4 \mathrm{Ex} 1)$ & $121.7 \pm 11.2$ & $30.5 \pm 2.6$ \\
$\mathrm{~A} 1+\gamma-4(\gamma-2 \mathrm{Ex} 1)$ & $70.2 \pm 4.4$ & $43.0 \pm 2.4$ \\
$\mathrm{~A} 1+\gamma-2(\gamma-4-\mathrm{Ex} 1 \mathrm{Ex2})$ & $39.5 \pm 3.6$ & $74.6 \pm 2.8$ \\
$\mathrm{~A} 1+\gamma-4(\gamma-2$-Ex1Ex2) & $84.8 \pm 10.4$ & $34.1 \pm 3.5$ \\
$\mathrm{~A} 1+\gamma-2(\gamma-4$ Ex2) & $63.1 \pm 3.8$ & $60.6 \pm 2.1$ \\
$\mathrm{~A} 1+\gamma-4(\gamma-2$ Ex2) & $89.3 \pm 10.4$ & $41.4 \pm 5.0$ \\
\hline
\end{tabular}

Mean ( \pm SEM) values from double $\mathrm{H}$-H-type fits to the individual data $(n=4-9$ patches per construct).

TARP $\gamma-2$ increases gammal to affect recovery from desensitized states. Furthermore, a 2-fold increase in gammal (from 7.6 to $15.0 \mathrm{~s}^{-1}$ ) mimicked a change in the recovery kinetics observed for TARP co-expression (Tables 3, 5; Fig. 9D).

We previously observed that TARP can accelerate the gating of AMPARs (Tomita et al., 2005), and this increase in gating kinetics is referred to as " $\beta$ ". Therefore, we decided to further model GluA1 with TARP $\gamma-2$ with respect to $\beta$. We observed that a 3.1 -fold increase in $\beta$ successfully replicated the onset of desensitization (Tables 3, 5; Fig. 9D). This result is consistent with the ability of TARPs to slow both desensitization and deactivation. To mimic the shape
Table 2. Time constants of desensitization (mean \pm SEM) for GluA1 coexpressed with various TARPs

\begin{tabular}{llrl}
\hline Desensitization decay & \multicolumn{1}{l}{$\tau_{1}$ (ms) } & \multicolumn{1}{c}{$\tau_{2}$ (ms) } & Slow component (\%) \\
\hline $\mathrm{A} 1$ & $2.0 \pm 0.1$ & $10.3 \pm 1.2$ & $10.2 \pm 3.2$ \\
$\mathrm{~A} 1+\gamma-2$ & $2.2 \pm 0.2$ & $7.6 \pm 0.6$ & $31.8 \pm 4.0$ \\
$\mathrm{~A} 1+\gamma-3$ & $2.1 \pm 0.1$ & $7.3 \pm 0.4$ & $38.0 \pm 4.5$ \\
$\mathrm{~A} 1+\gamma-4$ & $3.9 \pm 0.5$ & $18.0 \pm 1.9$ & $39.5 \pm 3.2$ \\
$\mathrm{~A} 1+\gamma-8$ & $3.1 \pm 0.1$ & $14.8 \pm 1.4$ & $43.2 \pm 4.3$ \\
$\mathrm{~A} 1 \_\gamma-2$ tandem & $3.8 \pm 0.9$ & $10.0 \pm 1.6$ & $37.8 \pm 4.5$ \\
$\mathrm{~A} 1 \_\gamma-3$ tandem & $3.6 \pm 0.2$ & $13.0 \pm 1.2$ & $40.7 \pm 9.4$ \\
$\mathrm{~A} 1+\gamma-2(\gamma-4 \mathrm{Ex} 1)$ & $4.5 \pm 0.8$ & $18.0 \pm 3.4$ & $40.0 \pm 3.1$ \\
$\mathrm{~A} 1+\gamma-4(\gamma-2 \mathrm{Ex} 1)$ & $2.9 \pm 0.3$ & $12.4 \pm 1.9$ & $44.1 \pm 7.2$ \\
$\mathrm{~A} 1+\gamma-2(\gamma-4 \mathrm{Ex} 1 \mathrm{Ex} 2)$ & $4.3 \pm 0.3$ & $16.0 \pm 0.6$ & $52.0 \pm 6.9$ \\
$\mathrm{~A} 1+\gamma-4(\gamma-2$ Ex1Ex2) & $2.3 \pm 0.2$ & $7.3 \pm 0.5$ & $32.0 \pm 2.9$ \\
$\mathrm{~A} 1+\gamma-2(\gamma-4 \mathrm{Ex} 2)$ & $3.4 \pm 0.3$ & $9.9 \pm 0.7$ & $31.0 \pm 2.3$ \\
$\mathrm{~A} 1+\gamma-4(\gamma-2 \mathrm{Ex} 2)$ & $3.4 \pm 0.2$ & $12.7 \pm 0.4$ & $37.4 \pm 3.3$ \\
\hline
\end{tabular}

Mean ( \pm SEM) values from biexponential fits to the individual data ( $n=4-11$ patches per construct).

of the recovery curve of GluA1 with TARP $\gamma-2$, a modest increase in $\gamma-2\left(35-50 \mathrm{~s}^{-1}\right)$ was required. $\gamma-2$ represents another rate constant of the recovery from desensitized states. Thus, the present data indicate that GluA1 recovers faster following a shorter pulse of glutamate, and TARP $\gamma-2$ accelerates both gating and recovery from desensitization.

\section{Discussion}

There are two factors which affect frequency-dependent glutamatergic synaptic transmission: the frequency of presynaptic glutamate release and the sensitivity of postsynaptic glutamate receptors to glutamate in short intervals. Recovery from 
A

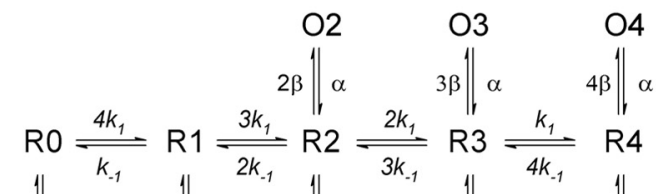

$$
\begin{aligned}
& \gamma_{0}\left\|4 \delta_{0}{ }^{k_{-1}} \gamma_{1}\right\| \delta_{1}{ }^{2 k_{-1}} \gamma_{1}\left\|2 \delta_{1}{ }^{3 k_{-1}} \gamma_{1}\right\| 3 \delta_{1}{ }^{4 k_{-1}} \gamma_{1} \| 4 \delta_{1}
\end{aligned}
$$

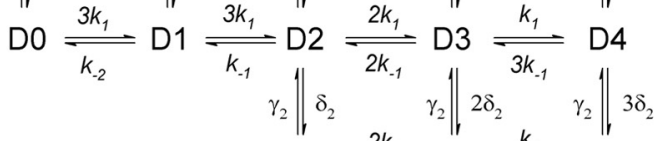

$$
\begin{aligned}
& \mathrm{D}_{2} 2 \stackrel{2 k_{1}}{\underset{k_{-1}}{\rightleftharpoons}} \mathrm{D}_{2} 3 \stackrel{k_{1}}{\underset{2 k_{-1}}{\rightleftharpoons}} \mathrm{D}_{2} 4
\end{aligned}
$$

\section{B}

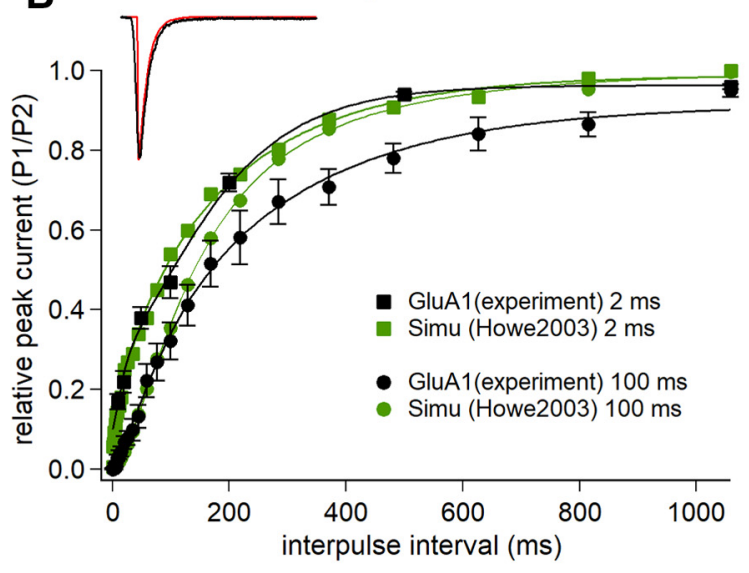

D

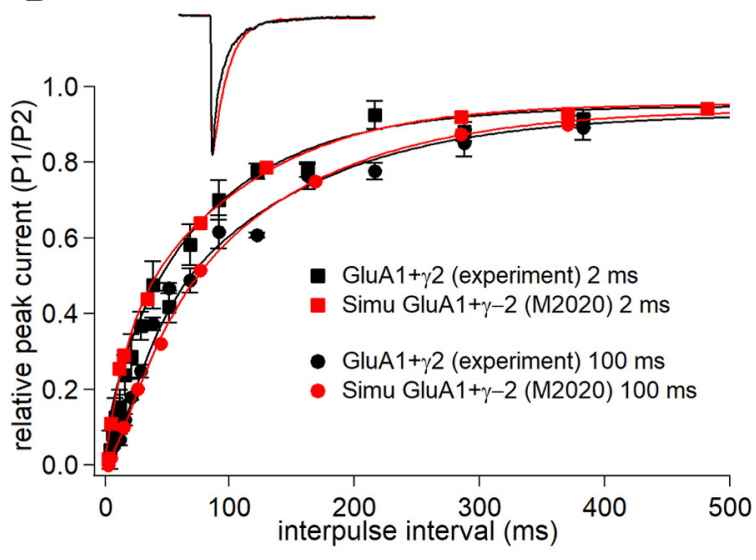

Figure 9. Kinetic models for GluA1 alone and GluA1 with TARPS. $\boldsymbol{A}$, A kinetic model for TARPless and TARPed AMPAR. This 16 state-model proposed by Robert and Howe (2003) incorporates multiple desensitized states. $\boldsymbol{B}-\boldsymbol{D}$, Simulated currents of recovery (colored dots) from brief $(2 \mathrm{~ms})$ and sustained (100 ms) applications of $10 \mathrm{~mm}$ glutamate are shown with experimental data (black) obtained from Figures 1, 2, 4. Simulated recovery kinetics of GluA1 according to the Howe 2003 model (B, green) and the 2020 Howe model (M2020; $\boldsymbol{C}$, red). $\boldsymbol{D}$, Simulated recovery of GluA1 with $\gamma-2$ (red) was estimated according to the M2020 model which had rate constants for $\gamma$ and $\beta$ increased, and was also compared with the experimental data from Figure 4 (black). The simulations performed with the M2020 model more closely approximate the experimental data.

Table 3. Rate constants for GluA1 and GluA1 with TARP $\gamma-2$ in Figure 9

\begin{tabular}{|c|c|c|c|}
\hline & $\begin{array}{l}\text { GluA1 } \\
\text { (Howe 2003) }\end{array}$ & $\begin{array}{l}\text { GluA1 } \\
\text { (M2020) }\end{array}$ & $\begin{array}{l}\text { GluA1+ } \gamma-2 \\
\text { (M2020) }\end{array}$ \\
\hline$\alpha$ & $3100 s^{-1}$ & $3100 s^{-1}$ & $3100 s^{-1}$ \\
\hline$\beta$ & $8000 s^{-1}$ & $8000 s^{-1}$ & $25000 s^{-1}$ \\
\hline$k_{1}$ & $2 \times 10^{7} \mathrm{~m}^{-1} / \mathrm{s}^{-1}$ & $2 \times 10^{7} \mathrm{~m}^{-1} / \mathrm{s}^{-1}$ & $2 \times 10^{7} \mathrm{~m}^{-1} / \mathrm{s}^{-1}$ \\
\hline$k_{-1}$ & $9000 s^{-1}$ & $9000 s^{-1}$ & $9000 s^{-1}$ \\
\hline$k_{2}$ & $910 \mathrm{M}^{-1} / \mathrm{S}^{-1}$ & $910 \mathrm{M}^{-1} / \mathrm{S}^{-1}$ & $910 \mathrm{M}^{-1} / \mathrm{S}^{-1}$ \\
\hline$k_{-2}$ & $0.41 \mathrm{~s}^{-1}$ & $1.5 \mathrm{~s}^{-1}$ & $1.5 \mathrm{~s}^{-1}$ \\
\hline$\delta_{0}$ & $3.3 \times 10^{-3} s^{-1}$ & $3.3 \times 10^{-3} s^{-1}$ & $3.3 \times 10^{-3} s^{-1}$ \\
\hline$\gamma_{0}$ & $1 s^{-1}$ & $1 s^{-1}$ & $1 s^{-1}$ \\
\hline$\delta_{1}$ & $1800 s^{-1}$ & $2500 \mathrm{~s}^{-1}$ & $2500 s^{-1}$ \\
\hline$\gamma_{1}$ & $7.6 s^{-1}$ & $7.6 s^{-1}$ & $15 s^{-1}$ \\
\hline$\delta_{2}$ & $200 s^{-1}$ & $50 s^{-1}$ & $50 s^{-1}$ \\
\hline$\gamma_{2}$ & $35 s^{-1}$ & $35 s^{-1}$ & $50 s^{-1}$ \\
\hline
\end{tabular}

The rate constants above refer to the kinetic model published by Robert and Howe (2003) and the simulation models used in the present study.

desensitization determines the time of a glutamate receptor's response to glutamate. Here, we tested the role of TARPs in the recovery of glutamate receptors. All four Type I TARP isoforms significantly reduced receptor-mediated paired-pulse depression and sped recovery from desensitization. Furthermore, in the presence of TARPs, recovery from desensitization was even more rapid and was modulated in an isoform-specific manner when brief synaptic-like applications of glutamate were applied. Our studies with TARP chimeras also indicate that sequence elements within both the first and second extracellular loops play important roles in TARP modulation of activation gating and recovery.

At short paired-pulse intervals, we anticipated that the effects of TARPs on activation gating would predominate and account for any quantitative differences between the isoforms. Following a short 2-ms application, the decay of the peak currents at the end of the glutamate application was faster than the rate of desensitization. However, because the receptors become desensitized from closed states, and all four subunits in the tetramer must lose glutamate before the receptors are desensitized, most receptors desensitize before the next brief application of glutamate (Raman and Trussell, 1995; Cho et al., 2007). TARPs selectively increase the apparent rate constant for receptorchannel opening to slow entry into desensitization, because on average, there are more openings before receptors enter stable, long-lived, desensitized states (Tomita et al., 2005; Cho et al., 2007). It was previously demonstrated that $\gamma-2$ and $\gamma-3$ mediate approximately a 2 -fold slowing in desensitization, whereas $\gamma-4$ and $\gamma-8$ mediate a 4 -fold slowing (Cho et al., 2007; Milstein et al., 2007). 
Table 4. Effects of each rate constant in modulating receptor kinetics

\begin{tabular}{|c|c|c|c|c|c|c|}
\hline & Rate constant change & Desensitization (ms) & Recovery time constant for $100 \mathrm{~ms}$ (ms) & Recovery time constant for $2 \mathrm{~ms}$ (ms) & Plateau/peak (\%) & $\overline{\text { Deactivation }(\mathrm{ms})}$ \\
\hline Howe 2003 & & 1.9 & 154 & 130 & 0.08 & 0.9 \\
\hline \multirow[t]{2}{*}{$\alpha$} & $10 \times$ higher & 0.35 & 148 & 110 & 0.00 & 0.13 \\
\hline & $10 \times$ lower & 15.2 & 152 & 88 & 0.63 & 9.2 \\
\hline \multirow[t]{2}{*}{$\beta$} & $10 \times$ higher & 10.8 & 150 & 71 & 0.54 & 5.8 \\
\hline & $10 \times$ lower & 0.5 & 149 & 91 & 0.00 & 0.3 \\
\hline \multirow[t]{2}{*}{$\kappa_{1}$} & $10 \times$ higher & 1.9 & 159 & 105 & 0.17 & 1.0 \\
\hline & $10 \times$ lower & 1.6 & 157 & 123 & 0.15 & 0.8 \\
\hline \multirow[t]{2}{*}{$\kappa_{-1}$} & $10 \times$ higher & 3.8 & 149 & 94 & 0.16 & 0.4 \\
\hline & $10 \times$ lower & 1.8 & $284^{\#}$ & $280^{\#}$ & 0.09 & 6.8 \\
\hline \multirow[t]{2}{*}{$\delta_{1}$} & $10 \times$ higher & 0.5 & $368^{\#}$ & $358^{\#}$ & 0.00 & 0.9 \\
\hline & $10 \times$ lower & 12.0 & 131 & 48 & 0.80 & 1.0 \\
\hline \multirow[t]{2}{*}{$\gamma_{1}$} & $10 \times$ higher & 1.8 & 24 & 15 & 1.02 & 0.94 \\
\hline & $10 \times$ lower & 1.8 & $953^{\#}$ & $825^{\#}$ & 0.00 & 0.93 \\
\hline \multirow[t]{2}{*}{$\delta_{2}$} & $10 \times$ higher & 1.9 & 163 & 134 & 0.00 & 0.92 \\
\hline & $10 \times$ lower & 1.8 & 144 & 131 & 0.79 & 0.95 \\
\hline \multirow{2}{*}{$\gamma_{2}$} & $10 \times$ higher & 1.8 & 138 & 91 & 0.76 & 0.94 \\
\hline & $10 \times$ lower & 1.8 & $470^{\#}$ & 149 & 0.00 & 0.93 \\
\hline \multirow[t]{2}{*}{$\kappa_{-2}$} & $10 \times$ higher & 1.9 & $123^{\#}$ & $113^{\#}$ & 0.05 & 0.93 \\
\hline & $10 \times$ lower & 1.9 & 149 & 124 & 0.03 & 0.93 \\
\hline \multirow[t]{2}{*}{$\gamma_{0}$} & $10 \times$ higher & 2.0 & 154 & 93 & 0.08 & 0.93 \\
\hline & $10 \times$ lower & 2.2 & 128 & 80 & 0.03 & 0.93 \\
\hline
\end{tabular}

\# indicates $R$ values $<0.95$ to fit to double $\mathrm{H}$-H-type equation.

Bold indicate changes in direction that are consistent with TARP-mediated modulation.

Table 5. Comparisons of models and empirical values

\begin{tabular}{|c|c|c|c|c|c|}
\hline & Desensitization (ms) & Recovery from 100 ms (ms) & Recovery from 2 ms (ms) & Plateau/peak (\%) & Deactivation (ms) \\
\hline GluA1(exp.) & $2.7 \pm 0.2$ & $167 \pm 20$ & $127 \pm 9.9$ & $0.4 \pm 0.1$ & $0.9 \pm 0.2$ \\
\hline Howe 2003 & 2.0 & 155 & 130 & 0.08 & 0.9 \\
\hline M2020 & 1.9 & 164 & 136 & 0.35 & 0.9 \\
\hline $\mathrm{M} 2020+\gamma-2$ & 4.1 & 99.4 & 77 & 2.4 & 1.8 \\
\hline GluA1+TARP $\gamma-2$ (exp.) & $5.0 \pm 0.6$ & $98.8 \pm 5.2$ & $79.7 \pm 5.0$ & $3.5 \pm 0.8$ & $1.7 \pm 0.3$ \\
\hline
\end{tabular}

Values estimated from biexponential fits (desensitization and deactivation) and the double $\mathrm{H}$-H-type equation fit (recovery from 100 or $2 \mathrm{~ms}$ ).

Interestingly, paired-pulse depression was no longer evident at an interpulse interval of $500 \mathrm{~ms}$, a time when recovery from desensitization is incomplete for GluA1 alone (Fig. 1). As previously described, recombinant receptors are formed from four core subunits (GluA1-4) and they recover from desensitization in two phases: an early phase that follows a sigmoidal time course, and a slower exponential phase which proceeds with a time constant of seconds (Robert and Howe, 2003). It has been demonstrated that GluA1 homomeric receptors account for $80-90 \%$ of the early phase of recovery. In addition, the early phase appears to be determined by two sequential rate-limiting steps. These steps may correspond to reassembly of the monomer-monomer interface in each of the two dimers (Sun et al., 2002; Robert and Howe, 2003; Armstrong et al., 2006). Possible structural correlates of the later exponential phase remain unknown. However, the desensitized states which give rise to the slow phase of recovery do not appear to be populated during a brief 2-ms pulse of glutamate, since paired-pulse depression is undetectable at $500 \mathrm{~ms}$.

Similar to TARP modulation of activation gating (Cho et al., 2007; Milstein et al., 2007; Jackson et al., 2011), the effects of the four TARPs on recovery from desensitization differed, with $\gamma-2$ and $\gamma-3$, and $\gamma-4$ and $\gamma-8$, behaving similarly (at least at long intervals). The faster recovery observed with the latter two TARPs accounts for the greater reductions in paired-pulse depression observed for these two isoforms at short intervals. For example, $\gamma-4$ produced least paired-pulse depression, a condition consistent with $\gamma-4$ exhibiting faster recovery compared with the other TARPs.
In agreement with previous studies, recovery from desensitization was faster with brief application protocols, and relative peak currents decreased with longer initial application times (Raman and Trussell, 1995; Robert and Howe, 2003; Zhang et al., 2006). These results suggest the existence of multiple desensitization states, and that increases in the rate of receptor escape from the first desensitization state increase with TARP co-expression. These results also suggest that TARPs speed the fast components of recovery to a greater extent following a brief application of glutamate. However, the proportion of the fast component of recovery remains stable. Despite the potential for TARPs to increase the rate of receptor escape from desensitization, we observed that the receptors eventually entered a desensitization state with increased duration of glutamate application.

Structural biological studies have revealed that the Ex1 domain slows AMPA receptor desensitization by interacting with the AMPAR LBD domain and stabilizing channels in an open state (Twomey et al., 2016; Zhao et al., 2016; Ben-Yaacov et al., 2017). In our previous studies, isoform-specificity was shown to contribute to TARP modulation of AMPAR gating. Specifically, the Ex1 domain accounted for differences in the modulation of desensitization and deactivation by TARPs (Tomita et al., 2005; Cho et al., 2007). Recovery from desensitization is associated with dissociation of receptors from glutamate and subunit rearrangements. This process is accelerated on TARP binding. In contrast to Exl modulation of activation gating, we demonstrated that exchange of the Ex1 domain alone did not invert the recovery phenotypes of $\gamma-2$ and $\gamma-4$. While our results do not 
exclude a role for the Ex1 domain in TARP modulation of recovery, they do indicate that other TARP regions contribute to the isoform-specific differences we report here. In fact, in another study, it was demonstrated that TARPs functionally modulate AMPA receptors, even when interactions between Ex1 and LBD were disrupted. Taken together, these results suggest that other interactions exist between TARPs and AMPA receptors (Dawe et al., 2016).

In addition to Ex1 modulating AMPA receptor gating, recent studies have proposed that Ex2 also interacts with AMPA receptors and modulates their gating (Zhao et al., 2016; Ben-Yaacov et al., 2017; Riva et al., 2017; Twomey et al., 2016). Here, we swapped the Ex2 domain of $\gamma-2$ and $\gamma-4$ and tested the recovery of these chimeras. We observed that the recovery profiles of $\gamma-2$ and $\gamma-4$ were reproduced following a sustained application of glutamate. These results suggest that the Ex2 domain is a key site for modulation of recovery function by TARPs. Moreover, the results from our Ex2 domain chimera suggest that the Ex2 domain determines the proportion of the channels which escape from various desensitization states. Our previous study showed that desensitization promotes the functional decoupling of TARPs from AMPA receptors (Morimoto-Tomita et al., 2009). Other studies have revealed that TARPs stabilize the open state, with Ex2 interacting with a LBD-TMD linker (Ben-Yaacov et al., 2017; Riva et al., 2017). These results, in combination with those of the present study, suggest that Ex 2 can easily interact with the LBD-TMD linker because of its close spatial proximity, and this would account for the observed isoform-specific modulation of recovery.

The frequency and shape of EPSCs are determined by several factors: presynaptic glutamate release, rate of glutamate clearance, and postsynaptic glutamate receptor properties. Clearly, the shapes of EPSCs are controlled by desensitization and deactivation, and thus could be modulated by TARPs. The postsynaptic AMPA receptor response to glutamate, as well as the rate of presynaptic glutamate release, are key factors for the frequency of EPSCs. In the presence of TARP expression, AMPA receptors exhibit faster recovery from desensitization in an isoform-specific manner at synapses responding to frequent glutamate release. In addition, we observed that TARPs are able to modulate the recovery of AMPARs in milliseconds, especially the fast component, which supports a role for AMPA receptors in achieving fast synaptic transmission.

In conclusion, the ability of TARPs to affect deactivation and desensitization of AMPA receptor is accompanied by a capacity to reduce receptor-mediated paired-pulse depression and hasten recovery from desensitization in an isoform-specific manner. The fast component of recovery was $\sim 4$ - to 5 -fold faster in the presence of TARPs compared with in their absence, especially for repeated brief applications of glutamate. Our TARP chimera results further indicate that sequence elements within the second extracellular loop play an important role in TARP modulation of recovery. Consequently, it is predicted that TARP isoform-specific modulations impact frequency-dependent synaptic signaling in the mammalian CNS.

\section{References}

Armstrong N, Jasti J, Beich-Frandsen M, Gouaux E (2006) Measurement of conformational changes accompanying desensitization in an ionotropic glutamate receptor. Cell 127:85-97.

Ben-Yaacov A, Gillor M, Haham T, Parsai A, Qneibi M, Stern-Bach Y (2017) Molecular mechanism of AMPA receptor modulation by TARP/ Stargazin. Neuron 93:1126-1137.e4.
Bredt DS, Nicoll RA (2003) AMPA receptor trafficking at excitatory synapses. Neuron 40:361-379.

Carbone AL, Plested AJ (2016) Superactivation of AMPA receptors by auxiliary proteins. Nat Commun 7:10178.

Chen C, Blitz DM, Regehr WG (2002) Contributions of receptor desensitization and saturation to plasticity at the retinogeniculate synapse. Neuron 33:779-788.

Chen L, Chetkovich DM, Petralia RS, Sweeney NT, Kawasaki Y, Wenthold RJ, Bredt DS, Nicoll RA (2000) Stargazin regulates synaptic targeting of AMPA receptors by two distinct mechanisms. Nature 408:936-943.

Cho CH, St-Gelais F, Zhang W, Tomita S, Howe JR (2007) Two families of TARP isoforms that have distinct effects on the kinetic properties of AMPA receptors and synaptic currents. Neuron 55:890-904.

Coombs ID, Cull-Candy SG (2009) Transmembrane AMPA receptor regulatory proteins and AMPA receptor function in the cerebellum. Neuroscience 162:656-665.

Dawe GB, Musgaard M, Aurousseau MRP, Nayeem N, Green T, Biggin PC, Bowie D (2016) Distinct structural pathways coordinate the activation of AMPA receptor-auxiliary subunit complexes. Neuron 89:1264-1276.

Devi SP, Howe JR, Auger C (2016) Train stimulation of parallel fibre to Purkinje cell inputs reveals two populations of synaptic responses with different receptor signatures. J Physiol 594:3705-3727.

DiGregorio DA, Rothman JS, Nielsen TA, Silver RA (2007) Desensitization properties of AMPA receptors at the cerebellar mossy fiber granule cell synapse. J Neurosci 27:8344-8357.

Dingledine R, Borges K, Bowie D, Traynelis SF (1999) The glutamate receptor ion channels. Pharmacol Rev 51:7-61.

Erreger K, Chen PE, Wyllie DJ, Traynelis SF (2004) Glutamate receptor gating. Crit Rev Neurobiol 16:187-224.

Fukaya M, Yamazaki M, Sakimura K, Watanabe M (2005) Spatial diversity in gene expression for VDCCgamma subunit family in developing and adult mouse brains. Neurosci Res 53:376-383.

Hashimoto K, Fukaya M, Qiao X, Sakimura K, Watanabe M, Kano M (1999) Impairment of AMPA receptor function in cerebellar granule cells of ataxic mutant mouse stargazer. J Neurosci 19:6027-6036.

Hollmann M, Heinemann S (1994) Cloned glutamate receptors. Annu Rev Neurosci 17:31-108.

Howe JR (2015) Modulation of non-NMDA receptor gating by auxiliary subunits. J Physiol 593:61-72.

Jackson AC, Nicoll RA (2011) The expanding social network of ionotropic glutamate receptors: TARPs and other transmembrane auxiliary subunits. Neuron 70:178-199.

Jackson AC, Milstein AD, Soto D, Farrant M, Cull-Candy SG, Nicoll RA (2011) Probing TARP modulation of AMPA receptor conductance with polyamine toxins. J Neurosci 31:7511-7520.

Jonas P, Major G, Sakmann B (1993) Quantal components of unitary EPSCs at the mossy fibre synapse on CA3 pyramidal cells of rat hippocampus. J Physiol 472:615-663.

Kato AS, Siuda ER, Nisenbaum ES, Bredt DS (2008) AMPA receptor subunit-specific regulation by a distinct family of type II TARPs. Neuron 59:986-996.

Kato AS, Gill MB, Yu H, Nisenbaum ES, Bredt DS (2010) TARPs differentially decorate AMPA receptors to specify neuropharmacology. Trends Neurosci 33:241-248.

Körber C, Werner M, Kott S, Ma ZL, Hollmann M (2007) The transmembrane AMPA receptor regulatory protein gamma 4 is a more effective modulator of AMPA receptor function than stargazin (gamma 2). J Neurosci 27:8442-8447.

Kott S, Werner M, Körber C, Hollmann M (2007) Electrophysiological properties of AMPA receptors are differentially modulated depending on the associated member of the TARP family. J Neurosci 27:3780-3789.

Lu HW, Balmer TS, Romero GE, Trussell LO (2017) Slow AMPAR synaptic transmission Is determined by stargazin and glutamate transporters. Neuron 96:73-80.e4.

McGee TP, Bats C, Farrant M, Cull-Candy SG (2015) Auxiliary subunit GSG1L acts to suppress calcium-permeable AMPA receptor function. J Neurosci 35:16171-16179.

Milstein AD, Nicoll RA (2009) TARP modulation of synaptic AMPA receptor trafficking and gating depends on multiple intracellular domains. Proc Natl Acad Sci USA 106:11348-11351. 
Milstein AD, Zhou W, Karimzadegan S, Bredt DS, Nicoll RA (2007) TARP subtypes differentially and dose-dependently control synaptic AMPA receptor gating. Neuron 55:905-918.

Morimoto-Tomita M, Zhang W, Straub C, Cho CH, Kim KS, Howe JR, Tomita S (2009) Autoinactivation of neuronal AMPA receptors via glutamate-regulated TARP interaction. Neuron 61:101-112.

Partin KM, Fleck MW, Mayer ML (1996) AMPA receptor flip/flop mutants affecting deactivation, desensitization, and modulation by cyclothiazide, aniracetam, and thiocyanate. J Neurosci 16:6634-6647.

Patneau DK, Mayer ML (1991) Kinetic analysis of interactions between kainate and AMPA: evidence for activation of a single receptor in mouse hippocampal neurons. Neuron 6:785-798.

Priel A, Kolleker A, Ayalon G, Gillor M, Osten P, Stern-Bach Y (2005) Stargazin reduces desensitization and slows deactivation of the AMPAtype glutamate receptors. J Neurosci 25:2682-2686.

Raman IM, Trussell LO (1992) The kinetics of the response to glutamate and kainate in neurons of the avian cochlear nucleus. Neuron 9:173-186.

Raman IM, Trussell LO (1995) The mechanism of alpha-amino-3-hydroxy5-methyl-4-isoxazolepropionate receptor desensitization after removal of glutamate. Biophys J 68:137-146.

Riva I, Eibl C, Volkmer R, Carbone AL, Plested AJ (2017) Control of AMPA receptor activity by the extracellular loops of auxiliary proteins. Elife 6 .

Robert A, Howe JR (2003) How AMPA receptor desensitization depends on receptor occupancy. J Neurosci 23:847-858.

Robert A, Irizarry SN, Hughes TE, Howe JR (2001) Subunit interactions and AMPA receptor desensitization. J Neurosci 21:5574-5586.

Robert A, Armstrong N, Gouaux JE, Howe JR (2005) AMPA receptor binding cleft mutations that alter affinity, efficacy, and recovery from desensitization. J Neurosci 25:3752-3762.

Schwenk J, Harmel N, Zolles G, Bildl W, Kulik A, Heimrich B, Chisaka O, Jonas P, Schulte U, Fakler B, Klocker N (2009) Functional proteomics identify cornichon proteins as auxiliary subunits of AMPA receptors. Science 323:1313-1319.

Schwenk J, Harmel N, Brechet A, Zolles G, Berkefeld H, Müller CS, Bildl W, Baehrens D, Hüber B, Kulik A, Klöcker N, Schulte U, Fakler B (2012) High-resolution proteomics unravel architecture and molecular diversity of native AMPA receptor complexes. Neuron 74:621-633.

Shanks NF, Savas JN, Maruo T, Cais O, Hirao A, Oe S, Ghosh A, Noda Y, Greger IH, Yates JR 3rd, Nakagawa T (2012) Differences in AMPA and kainate receptor interactomes facilitate identification of AMPA receptor auxiliary subunit GSG1L. Cell Rep 1:590-598.
Sobolevsky AI (2015) Structure and gating of tetrameric glutamate receptors. J Physiol 593:29-38.

Sun Y, Olson R, Horning M, Armstrong N, Mayer M, Gouaux E (2002) Mechanism of glutamate receptor desensitization. Nature 417:245-253.

Tomita S, Chen L, Kawasaki Y, Petralia RS, Wenthold RJ, Nicoll RA, Bredt DS (2003) Functional studies and distribution define a family of transmembrane AMPA receptor regulatory proteins. J Cell Biol 161:805-816.

Tomita S, Fukata M, Nicoll RA, Bredt DS (2004) Dynamic interaction of stargazin-like TARPs with cycling AMPA receptors at synapses. Science 303:1508-1511

Tomita S, Adesnik H, Sekiguchi M, Zhang W, Wada K, Howe JR, Nicoll RA, Bredt DS (2005) Stargazin modulates AMPA receptor gating and trafficking by distinct domains. Nature 435:1052-1058.

Traynelis SF, Wollmuth LP, McBain CJ, Menniti FS, Vance KM, Ogden KK, Hansen KB, Yuan H, Myers SJ, Dingledine R (2010) Glutamate receptor ion channels: structure, regulation, and function. Pharmacol Rev 62:405496.

Trussell LO, Fischbach GD (1989) Glutamate receptor desensitization and its role in synaptic transmission. Neuron 3:209-218.

Trussell LO, Zhang S, Raman IM (1993) Desensitization of AMPA receptors upon multiquantal neurotransmitter release. Neuron 10:1185-1196.

Turetsky D, Garringer E, Patneau DK (2005) Stargazin modulates native AMPA receptor functional properties by two distinct mechanisms. J Neurosci 25:7438-7448

Twomey EC, Yelshanskaya MV, Grassucci RA, Frank J, Sobolevsky AI (2016) Elucidation of AMPA receptor-stargazin complexes by cryo-electron microscopy. Science 353:83-86.

Vyklicky L Jr, Patneau DK, Mayer ML (1991) Modulation of excitatory synaptic transmission by drugs that reduce desensitization at AMPA/kainate receptors. Neuron 7:971-984.

Yan D, Tomita S (2012) Defined criteria for auxiliary subunits of glutamate receptors. J Physiol 590:21-31.

Zhang W, Robert A, Vogensen SB, Howe JR (2006) The relationship between agonist potency and AMPA receptor kinetics. Biophys J 91:1336-1346.

Zhang W, Devi SP, Tomita S, Howe JR (2014) Auxiliary proteins promote modal gating of AMPA- and kainate-type glutamate receptors. Eur J Neurosci 39:1138-1147.

Zhao Y, Chen S, Yoshioka C, Baconguis I, Gouaux E (2016) Architecture of fully occupied GluA2 AMPA receptor-TARP complex elucidated by cryo-EM. Nature 536:108-111. 